\title{
Motives with modulus, II: Modulus sheaves with transfers for proper modulus pairs
}

\author{
Bruno Kahn, Hiroyasu Miyazaki, Shuji Saito, and Takao Yamazaki
}

\begin{abstract}
We develop a theory of sheaves and cohomology on the category of proper modulus pairs. This complements [KMSY21], where a theory of sheaves and cohomology on the category of non-proper modulus pairs has been developed.
\end{abstract}

Keywords. modulus pair; presheaf with transfers; cd-structure

2020 Mathematics Subject Classification. 19E15; 14F42; 19D45; 19F15

[Français]

Motifs avec modules, II: faisceaux avec transferts pour les couples modulaires propres

Résumé. Nous présentons une théorie de faisceaux sur la catégorie des couples modulaires propres, et décrivons leur cohomologie. Ceci complète [KMSY21], où a été présentée une théorie de faisceaux sur la catégorie des couples modulaires non propres.

Received by the Editors on December 17, 2019, and in final form on November 26, 2020.

Accepted on December 19, 2020.

Bruno Kahn

IMJ-PRG, Case 247, 4 place Jussieu, 75252 Paris Cedex 05, France

e-mail: bruno.kahn@imj-prg.fr

Hiroyasu Miyazaki

RIKEN iTHEMS, Wako, Saitama 351-0198, Japan

e-mail: hiroyasu.miyazaki@riken.jp

Shuji Saito

Graduate School of Mathematical Sciences, University of Tokyo, 3-8-1 Komaba, Tokyo 153-8941, Japan

e-mail: sshuji@msb.biglobe.ne.jp

Takao Yamazaki

Institute of Mathematics, Tohoku University, Aoba, Sendai 980-8578, Japan

e-mail: ytakao@math.tohoku.ac.jp

The first author acknowledges the support of Agence Nationale de la Recherche (ANR) under reference ANR-12-BL01-0005. The work of the second author is supported by RIKEN iTHEMS, and by JSPS KAKENHI Grant (19K23413). The third author is supported by JSPS KAKENHI Grant (15H03606). The fourth author is supported by JSPS KAKENHI Grant (15K04773).

(c) by the author(s)

This work is licensed under http://creativecommons.org/licenses/by-sa/4.0/ 


\section{Contents}

Introduction . . . . . . . . . . . . . . . . . . . . . . . . . . . . . . . . . 2

1. Review of presheaf theory on modulus pairs . . . . . . . . . . . . . . . . . . . 5

2. Review of sheaf theory on non-proper modulus pairs . . . . . . . . . . . . . . . 7

3. A cd-structure on MSm . . . . . . . . . . . . . . . . . . . . . . . . . . . 10

4. Sheaves on MSm and MCor . . . . . . . . . . . . . . . . . . . . . . . . . 12

5. Cohomology in MNST . . . . . . . . . . . . . . . . . . . . . . . . . . . 15

6. Relation with NST . . . . . . . . . . . . . . . . . . . . . . . . . . . . . 21

7. Passage to derived categories . . . . . . . . . . . . . . . . . . . . . . . . . 24

Appendix: Categorical toolbox, II . . . . . . . . . . . . . . . . . . . . . . . . 27

References. . . . . . . . . . . . . . . . . . . . . . . . . 30

\section{Introduction}

This is a sequel to [KMSY21], where a theory of sheaves and cohomology on the category $\underline{\text { MCor of }}$ non-proper modulus pairs has been developed. This paper complements it by using work from [KM20] and [Miy20] to develop a theory of sheaves and cohomology on the category MCor of proper modulus pairs. This completes the repairs to the mistake in [KSY15]. The basic aim of both works is to lay a foundation for a theory of motives with modulus, to be completed in [KMSY20], generalizing Voevodsky's theory of motives in order to capture non $\mathbf{A}^{1}$-invariant phenomena.

In [KMSY21], Voevodsky's category Cor of finite correspondences on smooth separated schemes of finite type over a fixed base field $k$, was enlarged to the larger category of (non-proper) modulus pairs, MCor. Objects are pairs $M=\left(\bar{M}, M^{\infty}\right)$ consisting of a separated $k$-scheme of finite type $\bar{M}$ and an effective (possibly empty) Cartier divisor $M^{\infty}$ on it such that the complement $M^{\circ}:=\bar{M} \backslash M^{\infty}$ is in Sm (we call it the smooth interior). The group $\underline{\operatorname{MCor}}(M, N)$ of morphisms is defined as the subgroup of $\operatorname{Cor}\left(M^{\circ}, N^{\circ}\right)$ consisting of finite correspondences between smooth interiors whose closures in $\bar{M} \times_{k} \bar{N}$ are $\operatorname{proper}^{1}$ over $\bar{M}$ and satisfy

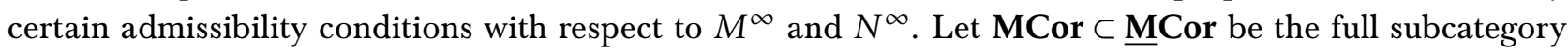
consisting of objects $\left(\bar{M}, M^{\infty}\right)$ with $\bar{M}$ proper over $k$.

We then define MPST (resp. MPST) as the category of additive presheaves of abelian groups on $\underline{\text { MCor }}$ (resp. MCor). We have a pair of adjunctions

$$
\operatorname{MPST} \underset{\tau_{!}}{\stackrel{\tau^{*}}{\longrightarrow}} \underline{\text { MPST, }}
$$

where $\tau^{*}$ is induced by the inclusion $\tau:$ MCor $\rightarrow$ MCor and $\tau_{!}$is its left Kan extension (see Lemma 1.2.3).

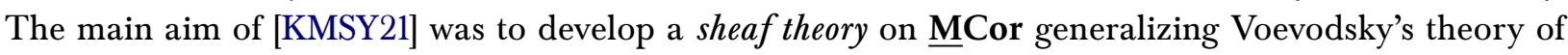
sheaves on Cor.

\footnotetext{
${ }^{1}$ Here we stress that we do not assume it is finite over $\bar{M}$.
} 
Definition 1. We define MNST to be the full subcategory of MPST of objects $F$ such that $F_{M}$ is a Nisnevich sheaf on $\bar{M}$ for every $M=\left(\bar{M}, M^{\infty}\right) \in \underline{\text { MCor, where } F_{M}}$ is the presheaf on $\bar{M}_{\text {Nis }}$ which associates $F\left(U, M^{\infty} \times \bar{M} U\right)$ to an étale map $U \rightarrow \bar{M}$.

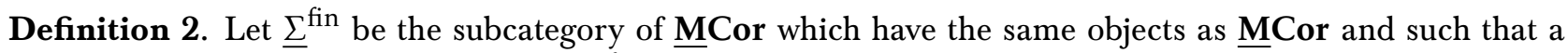
morphism $f \in \underline{\operatorname{MCor}}(M, N)$ belongs to $\underline{\Sigma}^{\text {fin }}$ if and only if $f^{\circ} \in \operatorname{Cor}\left(M^{\circ}, N^{\circ}\right)$ is the graph of an isomorphism $M^{\mathrm{o}} \simeq N^{\mathrm{o}}$ in $\mathrm{Sm}$ that extends to a proper morphism $\bar{f}: \bar{M} \rightarrow \bar{N}$ of $k$-schemes such that $M^{\infty}=\bar{f}^{*} N^{\infty}$.

Now the main result of [KMSY21] is the following.

Theorem 1 ([KMSY21, Theorem 2]). The following assertions hold.

(1) The inclusion $\underline{\mathrm{MNST}} \rightarrow \underline{\text { MPST }}$ has an exact left adjoint $\underline{a}_{\mathrm{Nis}}$ such that

$$
\left(\underline{a}_{\mathrm{Nis}} F\right)(M)=\underset{N \in \underline{\underline{\Sigma}}^{\mathrm{fin}} \downarrow M}{\lim }\left(F_{N}\right)_{\mathrm{Nis}}(N)
$$

for every $F \in \underline{\text { MPST }}$ and $M \in \underline{\text { MCor, where }}\left(F_{M}\right)_{\mathrm{Nis}}$ is the Nisnevich sheafification of the preshseaf $F_{M}$ on $\bar{M}_{\mathrm{Nis}}$. In particular $\underline{\mathrm{MNST}}$ is a Grothendieck abelian category.

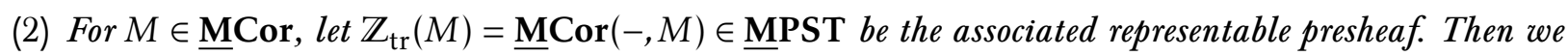

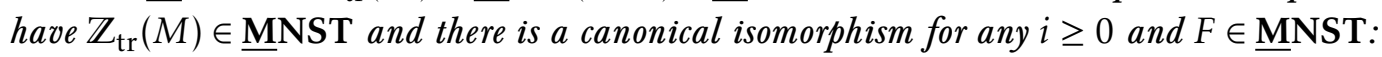

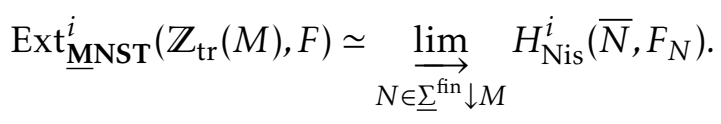

The aim of the present paper is to introduce a sheaf theory on MCor.

Definition 3. We define MNST to be the full subcategory of MPST of objects $F$ such that $\tau_{!} F \in \underline{\text { MNST }}$.

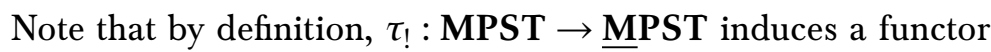

$$
\tau_{\mathrm{Nis}}: \text { MNST } \rightarrow \underline{\text { MNST }} .
$$

Now the main result of this paper is the following.

Theorem 2 (see Lemmas 4.2.2 and 4.2.5, Theorems 4.2.4, 5.1.1 and 5.1.3).

(1) We have $\tau^{*}(\underline{\mathrm{MNST}}) \subset \mathrm{MNST}$. Letting

$$
\tau^{\mathrm{Nis}}: \underline{\text { MNST }} \rightarrow \text { MNST }
$$

be the induced functor, the pair of adjoint functors $(*)$ induces a pair of adjoint functors

$$
\text { MNST } \underset{\tau_{\text {Nis }}}{\stackrel{\tau_{\text {Nis }}}{\leftrightarrows}} \text { MNST, }
$$

where $\tau_{\mathrm{Nis}}$ is exact and fully faithful. The functor $\tau^{\mathrm{Nis}}$ is also exact.

(2) The inclusion MNST $\rightarrow$ MPST has an exact left adjoint $a_{\mathrm{Nis}}$ such that $\underline{a}_{\mathrm{Nis}} \tau_{!}=\tau_{\mathrm{Nis}} a_{\mathrm{Nis}}$. In particular, MNST is a Grothendieck abelian category.

(3) For $M \in$ MCor, let $\mathbb{Z}_{\mathrm{tr}}(M)=\operatorname{MCor}(-, M) \in$ MPST be the associated representable presheaf. Then we have $\mathbb{Z}_{\mathrm{tr}}(M) \in$ MNST and there is a canonical isomorphism for any $i \geq 0$ and $F \in$ MNST:

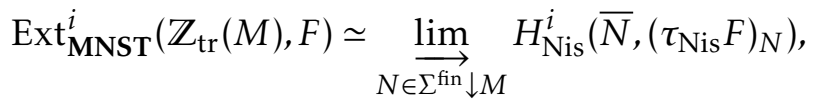

where $\Sigma^{\mathrm{fin}}:=\underline{\Sigma}^{\mathrm{fin}} \cap$ MCor. 
Finally we explain relations between cohomologies for MNST and NST. We denote by PST (resp. NST) Voevodsky's category of presheaves (resp. Nisnevich sheaves) with transfers. The functor given by $\omega:$ MCor $\rightarrow$ Cor, $\omega\left(\bar{M}, M^{\infty}\right)=\bar{M} \backslash\left|M^{\infty}\right|$ induces a pair of adjunctions

$$
\text { MPST } \underset{\stackrel{\omega_{1}}{\longrightarrow}}{\stackrel{\omega^{*}}{\longrightarrow}} \text { PST, }
$$

in a similar way as $(0.0 .1)$. (See $\S 6.2$ for details.)

Theorem 3 (see Proposition 6.2.1 and Theorem 6.3.2). The following assertions hold.

(1) We have

$$
\omega_{!}(\text {MNST }) \subset \text { NST } \quad \text { and } \quad \omega^{*}(\text { NST }) \subset \text { MNST } .
$$

The functors $\omega_{!}$and $\omega^{*}$ induce a pair of adjoint functors

$$
\text { MNST } \underset{\omega_{\text {Nis }}}{\stackrel{\omega^{\text {Nis }}}{\longrightarrow}} \text { NST, }
$$

such that

$$
\omega_{\mathrm{Nis}} a_{\mathrm{Nis}}=a_{\mathrm{Nis}}^{V} \omega_{!}, \quad \omega^{\mathrm{Nis}} a_{\mathrm{Nis}}^{V}=a_{\mathrm{Nis}} \omega^{*},
$$

where $a_{\mathrm{Nis}}^{V}:$ PST $\rightarrow$ NST is Voevodsky's sheafification functor. Moreover, $\omega^{\mathrm{Nis}}$ and $\omega_{\mathrm{Nis}}$ are exact and $\omega^{\text {Nis }}$ is fully faithful.

(2) For any $F \in$ MNST and $X \in \mathbf{S m}$ and $i \geq 0$, we have a canonical isomorphism

$$
H_{\mathrm{Nis}}^{i}\left(X, \omega_{\mathrm{Nis}} F\right) \simeq \underset{M \in \operatorname{MSm}(X)}{\lim _{\mathrm{Nis}}} H_{\mathrm{M}}^{i}\left(\bar{M}, F_{M}\right)
$$

where $\operatorname{MSm}(X)=\left\{M \in\right.$ MCor $\left.\mid M^{\mathrm{o}}=X\right\}$ is viewed as a cofiltered ordered set [KMSY21, Lemma 1.7.4].

A key ingredient of the proofs is Theorem 3.2.2, which is based on the works [Miy20] and [KM20].

\section{Acknowledgements}

Part of this work was done while the first author was visiting RIKEN iTHEMS under the invitation of the second author: the first and the second authors wish to thank both for their hospitality and excellent working conditions. Part of this work was done while the third author stayed at the university of Regensburg supported by the SFB grant "Higher Invariants". The third author is grateful to the support and hospitality received there. We also thank the referee for a thorough reading.

The first author thanks Joseph Ayoub for explaining him an easy but crucial result on unbounded derived categories (Lemma A.2.7).

\section{Notation and conventions}

In the whole paper we fix a base field $k$. Let Sch be the category of separated schemes of finite type over $k$, and let $\mathbf{S m}$ be its full subcategory of smooth schemes. We write Cor for Voevodsky's category of finite correspondences [Voe00].

An additive functor between additive categories is called strongly additive if it commutes with all representable direct sums. A Grothendieck topology is called subcanonical if every representable presheaf is a sheaf.

Let $\mathcal{C}$ and $\mathcal{D}$ be sites, and $u: \mathcal{C} \rightarrow \mathcal{D}$ a functor. We say that $u$ is continuous (resp. cocontinuous) if the functor $u^{*}: \hat{\mathcal{D}} \rightarrow \hat{\mathcal{C}}$ (resp. $u_{*}: \hat{\mathcal{C}} \rightarrow \hat{\mathcal{D}}$ ) between categories of presheaves carries sheaves to sheaves. ( $c f$. [SGA4, exposé III] and [KM20, §A.1].) 


\section{Review of presheaf theory on modulus pairs}

\subsection{Categories of modulus pairs}

A modulus pair $M$ consists of $\bar{M} \in \mathrm{Sch}$ and an effective Cartier divisor $M^{\infty} \subset \bar{M}$ such that the open subset $M^{\circ}:=\bar{M}-\left|M^{\infty}\right|$ is smooth over $k$. (The case $\left|M^{\infty}\right|=\emptyset$ is allowed.) We say that $M$ is proper if $\bar{M}$ is. Note that $\bar{M}$ is automatically reduced, and $M^{\circ}$ is dense in $\bar{M}$ [KMSY21, Remark 1.1.2 (3)].

Let $M_{1}, M_{2}$ be modulus pairs. Let $Z \in \operatorname{Cor}\left(M_{1}^{\mathrm{o}}, M_{2}^{\mathrm{o}}\right)$ be an elementary (i.e. integral) finite correspondence in the sense of Voevodsky [Voe00]. We write $\bar{Z}^{N}$ for the normalization of the closure $\bar{Z}$ of $Z$ in $\bar{M}_{1} \times \bar{M}_{2}$ and $p_{i}: \bar{Z}^{N} \rightarrow \bar{M}_{i}$ for the canonical morphisms for $i=1,2$. We say $Z$ is admissible (resp. left-proper) for $\left(M_{1}, M_{2}\right)$ if $p_{1}^{*} M_{1}^{\infty} \geq p_{2}^{*} M_{2}^{\infty}$ (resp. $\bar{Z}$ is proper over $\bar{M}_{1}$ ).

By [KMSY21, Proposition 1.2.3 and 1.2.6], modulus pairs and left proper admissible correspondences

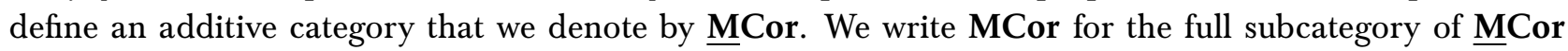
whose objects are proper modulus pairs.

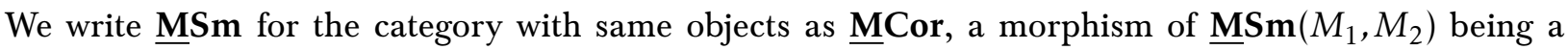
(scheme-theoretic) $k$-morphism $f^{\mathrm{o}}: M_{1}^{\mathrm{o}} \rightarrow M_{2}^{\mathrm{o}}$ whose graph belongs to $\underline{\operatorname{MCor}}\left(M_{1}, \bar{M}_{2}\right)$. We write $\mathbf{M S m}$ for the full subcategory of $\underline{\mathbf{M S m}}$ whose objects are proper modulus pairs.

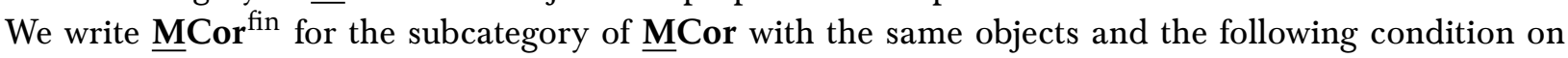
morphisms: $\alpha \in \underline{\operatorname{MCor}}(M, N)$ belongs to $\underline{\operatorname{MCor}}{ }^{\text {fin }}(M, N)$ if and only if, for any component $Z$ of $\alpha$, the projection $\bar{Z} \rightarrow \bar{M}$ is finite, where $\bar{Z}$ is the closure of $Z$ in $\bar{M} \times \bar{N}$. We write MCor ${ }^{\text {fin }}$ for the full subcategory

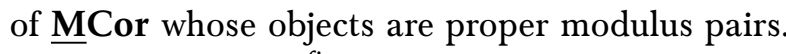

We write $\underline{M S m}^{\text {fin }}$ for the subcategory of $\underline{\mathrm{MSm}}$ with the same objects and such that a morphism $f: M \rightarrow N$ belongs to $\underline{\mathbf{M S m}}{ }^{\text {fin }}$ if and only if $f^{\mathrm{o}}: M^{\mathrm{o}} \rightarrow N^{\mathrm{o}}$ extends to a $k$-morphism $\bar{f}: \bar{M} \rightarrow \bar{N}$. We write $\mathbf{M S m}^{\text {fin }}$ for the full subcategory of $\underline{\mathbf{M S m}}$ whose objects are proper modulus pairs. A morphism $f: M \rightarrow N$ in $\underline{M S m}^{\text {fin }}$ is minimal if we have $f^{*} N^{\infty}=M^{\infty}$.

Remarks 1.1.1.

(1) For $M \in \underline{\mathbf{M S}} \mathbf{m}^{\text {fin }}$, set $M^{N}:=\left(\bar{M}^{N},\left.M^{\infty}\right|_{\bar{M}^{N}}\right)$ where $p: \bar{M}^{N} \rightarrow \bar{M}$ is the normalization and $\left.M^{\infty}\right|_{\bar{M}^{N}}$ is the pull-back of $M^{\infty}$ to $\bar{M}^{N}$. Then $p: M^{N} \rightarrow M$ is an isomorphism in $\underline{\text { MCor fin }}$ and $\underline{\text { MSm (but }}$ not in $\underline{\mathbf{M S}} \mathbf{m}^{\text {fin }}$ in general).

(2) Let $f: M \rightarrow N$ be a morphism in $\underline{\mathbf{M S}} \mathbf{m}^{\text {fin }}$. The reducedness of $\bar{M}$, the separatedness of $\bar{N}$ and the denseness of $M^{\mathrm{o}}$ in $\bar{M}$ imply that this extension $\bar{f}$ is unique. This yields a forgetful functor $\underline{M S m}^{\text {fin }} \rightarrow$ Sch, which sends $M$ to $\bar{M}$.

We have the following commutative diagram of inclusion functors

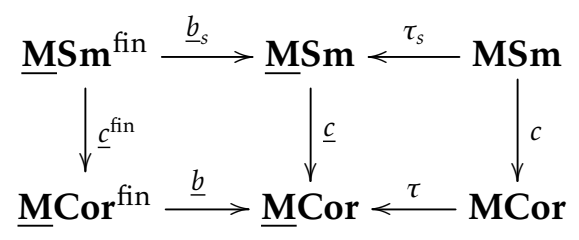

\subsection{Presheaves}

Definition 1.2.1. By a presheaf we mean here an additive contravariant functor to the category of abelian groups. (A functor is called additive if it commutes with finite coproducts.)

(1) The category of presheaves on MSm (resp. $\underline{\mathbf{M S m}}, \underline{\mathbf{M S m}}{ }^{\mathrm{fin}}$ ) is denoted by MPS (resp. $\underline{\text { MPS, }} \underline{\mathbf{M P S}}{ }^{\mathrm{fin}}$ ).

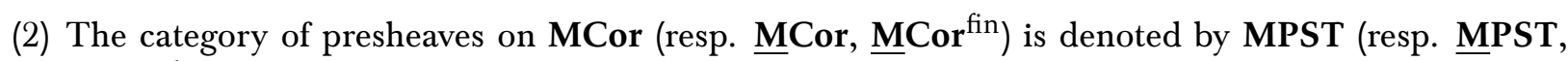
MPST $^{\text {fin }}$ ). 
(3) We write

$$
\begin{aligned}
& \mathbb{Z}_{\mathrm{tr}}: \underline{\text { MCor } \rightarrow} \text { MPST, } \quad \text { MCor } \rightarrow \text { MPST }, \\
& \mathbb{Z}_{\mathrm{tr}}^{\mathrm{fin}}: \underline{\text { MCor }}^{\mathrm{fin}} \rightarrow \underline{\text { MPST }}^{\text {fin }},
\end{aligned}
$$

for the associated representable presheaf functors.

(4) For $M \in \underline{\mathbf{M S m}}$, we denote by $\mathbb{Z}^{p}(M)$ the presheaf with values in abelian groups defined by

$$
\underline{\mathbf{M S m}} \ni N \mapsto \mathbb{Z} \underline{\mathbf{M S}} \mathbf{S}(N, M),
$$

where for any set $S$ we denote by $\mathbb{Z} S$ the free abelian group on $S$.

Diagram (1.1.1) induces a commutative diagram of functors on presheaf categories:

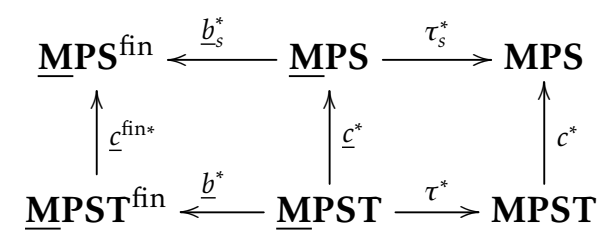

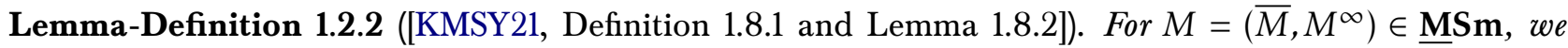
denote by $\operatorname{Comp}(M)$ the category whose objects are morphisms $M \stackrel{j_{N}}{\longrightarrow} N$ in $\underline{\mathbf{M S m}}{ }^{\text {fin }}$ such that

(i) $N \in \mathbf{M S m}$;

(ii) $\overline{j_{N}}: \bar{M} \rightarrow \bar{N}$ is a dense open immersion;

(iii) $j_{N}$ is minimal, i.e., $M^{\infty}={\overline{j_{N}}}^{*} N^{\infty}$;

(iv) we have $N^{\infty}=M_{N}^{\infty}+C$ for some effective Cartier divisors $M_{N}^{\infty}, C$ on $\bar{N}$ satisfying $\bar{N} \backslash|C|=j(\bar{M})$. (Note that, therefore, $M^{\infty}={\overline{j_{N}}}^{*} M_{N}^{\infty}$ )

For $N_{1}, N_{2} \in \operatorname{Comp}(M)$ we define

$$
\operatorname{Comp}(M)\left(N_{1}, N_{2}\right)=\left\{\gamma \in \operatorname{MSm}\left(N_{1}, N_{2}\right) \mid \gamma \circ j_{N_{1}}=j_{N_{2}}\right\} .
$$

The category $\operatorname{Comp}(M)$ is nonempty, ordered and cofltered.

Lemma 1.2.3 ([KMSY21, Proposition 2.4.1 and Lemma 2.4.2]).

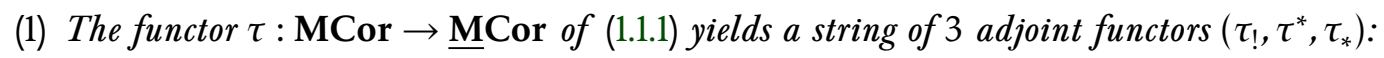

$$
\operatorname{MPST} \underset{\stackrel{\tau_{*}}{\longrightarrow}}{\stackrel{\tau_{!}^{*}}{\longrightarrow}} \text { MPST, }
$$

where $\tau_{!}, \tau_{*}$ are fully faithful, $\tau^{*}$ is a localisation and the adjunction map $\mathrm{Id} \rightarrow \tau^{*} \tau_{!}$is an isomorphism. The functors $\tau_{!}$and $\tau^{*}$ commute with all colimits and $\tau_{!}$has a pro-left adjoint represented by Comp, hence is exact.

(2) The same statements as (1) hold for the functor $\tau_{s}: \mathbf{M S m} \rightarrow \underline{\mathrm{MSm}}$.

(3) For $G \in$ MPST and $M \in \underline{\text { MSm, we have }}$

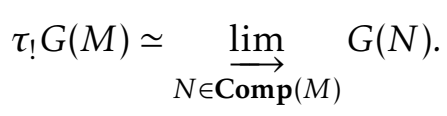

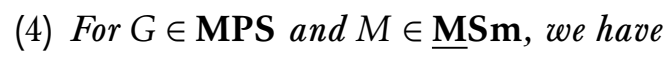

$$
\tau_{s !} G(M) \simeq \underset{N \in \operatorname{Comp}(M)}{\lim _{\longrightarrow}} G(N) .
$$


Lemma 1.2.4 ([KMSY21, Proposition 2.5.1]). Let $\underline{b}: \underline{\text { MCor }}^{\text {fin }} \rightarrow$ MCor be the inclusion functor from (1.1.1). Then $\underline{b}$ yields a string of 3 adjoint functors $\left(\underline{b}_{!}, \underline{b}^{*}, \underline{b}_{*}\right)$ :

$$
\underline{M P S T}^{\text {fin }} \underset{\stackrel{\underline{b}_{!}}{\stackrel{\underline{b}_{*}^{*}}{\longrightarrow}}}{\stackrel{\text { MPST, }}{\longrightarrow}}
$$

where $\underline{b}_{1}, \underline{b}_{*}$ are localisations; $\underline{b}^{*}$ is exact and fully faithful; $\underline{b}_{!}$has a pro-left adjoint, hence is exact. For

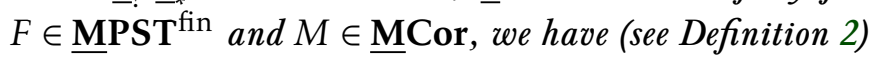

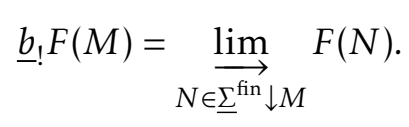

The same statements hold for $\underline{b}_{s}$ from (1.1.1).

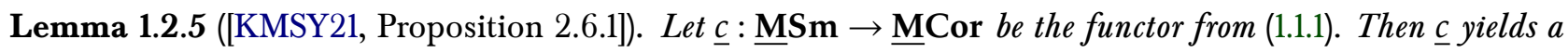
string of 3 adjoint functors $\left(\underline{c}_{1}, \underline{c}^{*}, \underline{c}_{*}\right)$ :

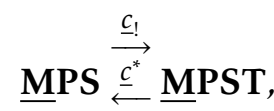

$$
\begin{aligned}
& \stackrel{\stackrel{c_{*}}{\longrightarrow}}{\longrightarrow}
\end{aligned}
$$

where $\underline{c}^{*}$ is exact and faithful (but not full). The same statements hold for $\underline{c}^{\text {fin }}$ and c from (1.1.1). We have

$$
\begin{gathered}
c^{*} \tau^{*}=\tau_{s}^{*} \underline{c}^{*}, \quad \underline{c}^{*} \tau_{!}=\tau_{s !} c^{*}, \\
\underline{c}^{\mathrm{fin} *} \underline{b}^{*}=\underline{b}_{s}^{*} \underline{c}^{*}, \quad \underline{b} ! \underline{c}_{!}^{\mathrm{fin}}=\underline{c} ! \underline{\underline{b}} \underline{s}_{s}, \quad \underline{c}^{*} \underline{b}_{!}=\underline{b}_{s} \underline{\mathrm{c}}^{\mathrm{fin} *} .
\end{gathered}
$$

For the readers' convenience, we recall the following lemma from [KMSY21, Lemma A.8.1]:

Lemma 1.2.6. Let $\mathcal{C}, \mathcal{D}$ be abelian categories and let $\mathcal{C}^{\prime} \subset \mathcal{C}, \mathcal{D}^{\prime} \subset \mathcal{D}$ be full abelian subcategories. Let $c: \mathcal{C} \rightarrow \mathcal{D}$ and $c^{\prime}: \mathcal{C}^{\prime} \rightarrow \mathcal{D}^{\prime}$ be additive functors satisfying $c i_{C}=i_{D} c^{\prime}$, where $i_{C}: \mathcal{C}^{\prime} \rightarrow \mathcal{C}$ and $i_{D}: \mathcal{D}^{\prime} \rightarrow \mathcal{D}$ are the inclusion functors.

(1) If c is faithful, so is $c^{\prime}$.

(2) Suppose that $i_{D}$ is strongly additive or has a strongly additive left inverse (for example, a left adjoint). If $c$ and $i_{C}$ are strongly additive, so is $c^{\prime}$.

(3) Suppose that $i_{C}$ has a left adjoint $a_{C}$. If $c$ has a left adjoint $d$, then $d^{\prime}=a_{C} d i_{D}$ is a left adjoint of $c^{\prime}$. If $d$ and $a_{\mathcal{C}}$ are exact, so is $d^{\prime}$. Moreover, $a_{\mathcal{C}} d=d^{\prime} a_{\mathcal{D}}$ if $i_{\mathcal{D}}$ has a left adjoint $a_{\mathcal{D}}$.

(4) Suppose that $i_{C}$ and $i_{D}$ have left adjoints $a_{C}$ and $a_{D}$, that $a_{D}$ is exact, and that $a_{D} c=c^{\prime} a_{C}$. If $c$ is exact, then so is $c^{\prime}$.

\section{Review of sheaf theory on non-proper modulus pairs}

In this section we recall some basic definitions and properties on sheaves on categories of non-proper modulus pairs from [KMSY21, §4.1 and §4.2].

Let Sq be the product category of $[0]=\{0 \rightarrow 1\}$ with itself, depicted as

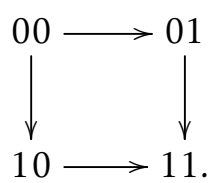

For any category $\mathcal{C}$, denote by $\mathcal{C}^{\mathbf{S q}}$ for the category of functors from Sq to $\mathcal{C}$. A functor $f: \mathcal{C} \rightarrow \mathcal{C}^{\prime}$ induces a functor $f^{\mathrm{Sq}}: \mathcal{C}^{\mathrm{Sq}} \rightarrow \mathcal{C}^{\prime \mathbf{S q}}$.

Let us consider $Q \in \underline{\mathbf{M S m}}^{\mathrm{Sq}}$. We write $Q(i j)=\left(\bar{Q}(i j), Q^{\infty}(i j)\right)$ for all $i, j \in\{0,1\}$. We also write $Q^{\mathrm{o}}(i j):=\bar{Q}(i j)-\left|Q^{\infty}(i j)\right|$. 


\subsection{The $\underline{M V}^{\text {fin }}$ cd-structure}

\section{Definition 2.1.1.}

(1) A Cartesian square

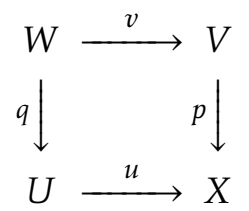

in Sch is called an elementary Nisnevich square if $p$ is étale and $p^{-1}(X \backslash U)_{\mathrm{red}} \rightarrow(X \backslash U)_{\mathrm{red}}$ is an isomorphism and if $u$ is an open embedding. In this situation, we say $U \sqcup V \rightarrow X$ is an elementary Nisnevich cover. Recall that an additive presheaf is a Nisnevich sheaf if and only if it transforms any elementary Nisnevich square into a cartesian square [Voe10a, Corollary 2.17], [Voe10b, Theorem 2.2].

(2) A diagram (2.1.1) in $\underline{\mathbf{M S m}}{ }^{\text {fin }}$ is called an $\underline{M V}^{\text {fin }}{ }_{\text {-square if it becomes an elementary Nisnevich square }}$ (in Sch) after replacing $X, U, V, W$ by $\bar{X}, \bar{U}, \bar{V}, \bar{W}(c f$. Remark 1.1.1(2)) and all morphisms are minimal.

Proposition 2.1.2 ([KMSY21, Proposition 3.2.3 (2)]). The cd-structure $P_{\underline{M V}}$ fin on $\underline{\mathbf{M S m}}{ }^{\text {fin }}$ consisting of $\underline{\mathrm{MV}}^{\text {fin }}$. squares is strongly complete and strongly regular, hence complete and regular in the sense of [Voe10a] (see [KMSY21, Definition A.7.1 and A.7.4]).

We define $\underline{M N S}^{\text {fin }}$ to be the full subcategory of $\underline{M P S}^{\text {fin }}$ consisting of sheaves with respect to the Grothendieck topology associated to $P_{\underline{M V}^{\text {fin }}}$. Let

$$
\underline{a}_{s, \text { Nis }}^{\text {fin }}: \underline{\text { MPS }}^{\text {fin }} \rightarrow \underline{\text { MNS }}^{\text {fin }}
$$

be the sheafification functor, that is, the left adjoint of the inclusion functor $\underline{i}_{s, N i s}^{\text {fin }}: \underline{\text { MNS }}{ }^{\text {fin }} \hookrightarrow \underline{\text { MPS }}{ }^{\text {fin }}$. It exists for general reasons and is exact [SGA4, exposé II, théorème 3.4].

Lemma 2.1.3 ([KMSY21, Lemma 3.1.3]). Let $M \in \underline{M S m}^{\text {fin }}$. Let $M_{\mathrm{Nis}}$ be the category of morphisms $f: N \rightarrow M$ in $\underline{M S m}^{\text {fin }}$ such that $\bar{f}$ is étale and $\bar{f}^{*} M^{\infty}=N^{\infty}$, endowed with the topology induced by $P_{\mathrm{MV}}{ }_{\text {fin }}$, and let $(\bar{M})_{\text {Nis }}$ be the (usual) small Nisnevich site on $\bar{M}$. Then we have an isomorphism of sites

$$
M_{\mathrm{Nis}} \rightarrow(\bar{M})_{\mathrm{Nis}}, \quad N \mapsto \bar{N},
$$

whose inverse is given by $(p: X \rightarrow \bar{M}) \mapsto\left(X, p^{*}\left(M^{\infty}\right)\right)$. (This isomorphism of sites depends on the choice of $M^{\infty}$.)

Notation 2.1.4. Let $M=\left(\bar{M}, M^{\infty}\right) \in \underline{\mathbf{M S m}}{ }^{\text {fin }}$ and $F \in \underline{M P S}^{\text {fin }}$. We write $F_{M}$ for the presheaf on $(\bar{M})_{\text {ét }}$ which associates $F\left(U, U \times \bar{M} M^{\infty}\right)$ to an étale map $U \rightarrow \overline{\bar{M}}$. By Lemma 2.1.3, $F \in \underline{\text { MNS }}{ }^{\text {fin }}$ if and only if $F_{M}$ is a sheaf on $(\bar{M})_{\text {Nis }}$ for every $M \in \underline{\mathbf{M S}} \mathbf{m}^{\text {fin }}$.

Let $\underline{\text { MNST }}^{\text {fin }}$ be the full subcategory of $\underline{\text { MPST }}^{\text {fin }}$ consisting of all objects $F \in \underline{M P S T}^{\text {fin }}$ such that $\underline{c}^{\text {fin* }} F \in \underline{M}^{\text {MNS }}{ }^{\text {fin }}$, where $\underline{c}^{\text {fin* }}: \underline{M P S T}^{\text {fin }} \rightarrow \underline{M P S}^{\text {fin }}$ is from (1.2.1).

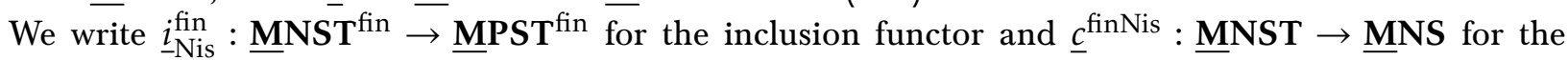
functor induced by $\underline{\underline{f i n}}^{\text {fin }}$. By definition, we have

$$
\underline{c}^{\mathrm{fin} *} \underline{i}_{\mathrm{Nis}}^{\mathrm{fin}}=\underline{i}_{s, \mathrm{Nis}} \underline{c}^{\mathrm{finNis}} .
$$

Theorem 2.1.5 ([KMSY21, Theorem 3.5.3]). The functor $\underline{i}_{\mathrm{Nis}}^{\mathrm{fin}}$ has an exact left adjoint

$$
\underline{a}_{\mathrm{Nis}}^{\mathrm{fin}}: \underline{\text { MPST }}^{\mathrm{fin}} \rightarrow \underline{\mathbf{M N S T}}^{\mathrm{fin}}
$$

satisfying

$$
\underline{c}^{\mathrm{finNis}} \underline{a}_{\mathrm{Nis}}^{\mathrm{fin}}=\underline{a}_{s \mathrm{Nis}} \underline{c}^{\mathrm{fin}}{ }^{\mathrm{fin}} .
$$


In particular $\underline{M N S T}^{\mathrm{fin}}$ is Grothendieck. Moreover, $\underline{\mathrm{MNST}}^{\mathrm{fin}}$ is closed under infinite direct sums in MPST $^{\mathrm{fin}}$ and the inclusion functor $\underline{i}_{\mathrm{Nis}}^{\mathrm{fin}}: \underline{\mathrm{MNST}}^{\mathrm{fin}} \rightarrow \underline{\mathrm{MPST}}^{\mathrm{fin}}$ is strongly additive.

\subsection{The $\underline{M V}$ cd-structure}

Definition 2.2.1 ([KMSY21, Definition 4.1.1]). A diagram in $\underline{\mathrm{MSm}}$ is called an $\underline{\mathrm{MV}}$-square if it is isomorphic in $\underline{\mathbf{M S m}} \mathbf{S}^{\mathbf{S q}}$ to $\underline{b}_{s}^{\mathbf{S q}}(Q)$ for some $\underline{\mathbf{M V}}^{\text {fin }}$-square $Q$ as in Definition 2.1.1, where $\underline{b}_{s}^{\text {Sq }}$ is the functor induced on squares by $\underline{b}_{s}: \underline{\mathbf{M S m}}{ }^{\text {fin }} \rightarrow \underline{\mathrm{MS}}$ m from (1.1.1). Let $\underline{P}_{\underline{\mathrm{MV}}}$ be a cd-structure on $\underline{\mathbf{M S m}}$ given by the collection of MV-squares.

Theorem 2.2.2 ([KMSY21, Theorem 4.1.2]). The cd-structure $P_{\mathrm{MV}}$ is strongly complete and strongly regular, in particular complete and regular (see [KMSY21, Definition A.7.1 and A.7.4]).

Remark 2.2.3. In view of Lemma 2.1.3, the topology defined by $P_{\underline{\mathrm{MV}}}$ in is subcanonical. This is also true for $P_{\text {MV }}$ by [Miy20, Theorem 4.5.1].

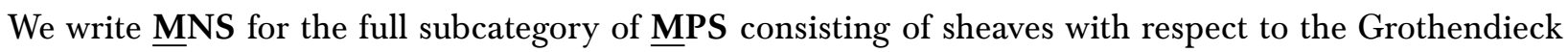
topology on $\underline{\mathrm{M} S m}$ associated to $\underline{P}_{\mathrm{MV}}$. We denote by $\underline{i}_{s, \mathrm{Nis}}: \underline{\mathrm{MNS}} \rightarrow \underline{\mathrm{MPS}}$ the inclusion functor.

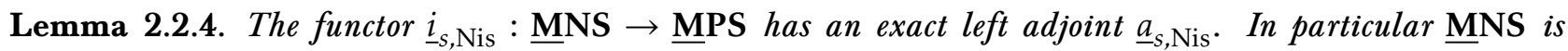
Grothendieck. Moreover, the following conditions are equivalent for $F \in \underline{\text { MPS. }}$

(i) $F \in \underline{\mathbf{M N S}}$.

(ii) It transforms any $\underline{\mathrm{MV}}^{\mathrm{fin}}$-square

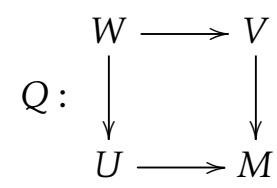

into an exact sequence

$$
0 \rightarrow F(M) \rightarrow F(U) \oplus F(V) \rightarrow F(W) .
$$

Proof. The first two assertions follow from the general properties of Grothendieck topologies [SGA4, exposé II]. The equivalence (i) $\Longleftrightarrow$ (ii) follows from [Voe10a, Corollary 2.17] in view of Theorem 2.2.2.

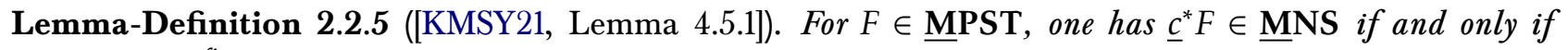
$\underline{b}^{*} F \in \underline{M N S T}^{\text {fin }}$, where

$$
\underline{b}^{*}: \underline{\text { MPST }} \rightarrow \underline{\text { MPST }}^{\mathrm{fin}}, \quad \underline{c}^{*}: \underline{\text { MPST }} \rightarrow \underline{\text { MPS }}
$$

are from (1.2.1).

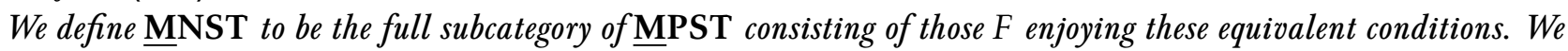

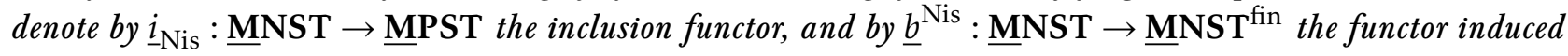
by $\underline{b}^{*}$.

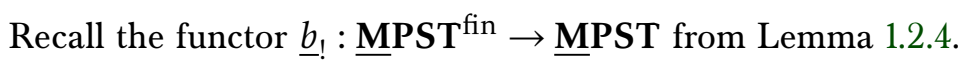

Proposition 2.2.6 ([KMSY21, Proposition 4.5.4]). We have $\underline{b}_{!}\left(\underline{M N S T}^{\mathrm{fin}}\right) \subset \underline{\mathrm{MNST}}$. We can thus consider $\underline{b}_{\mathrm{Nis}}: \underline{\mathrm{MNST}}^{\mathrm{fin}} \rightarrow \underline{\mathrm{MNST}}$, the restriction of $\underline{b}_{!}$, so that we have

$$
\underline{b}, \underline{i}_{\mathrm{Nis}}^{\mathrm{ifi}}=\underline{i}_{\mathrm{Nis}} \underline{b}_{\mathrm{Nis}} \text {. }
$$

Then $\underline{b}_{\mathrm{Nis}}$ is an exact left adjoint of $\underline{b}^{\mathrm{Nis}}$, and is fully faithful.

Theorem 2.2.7 ([KMSY21, Lemma 4.5.3, Theorem 4.5.5 and Proposition 4.5.6]). The category MNST contains

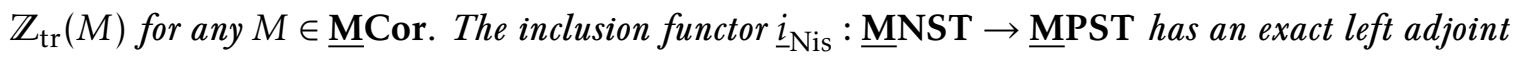

$$
\underline{a}_{\mathrm{Nis}}: \underline{\text { MPST }} \rightarrow \underline{\text { MNST }}
$$




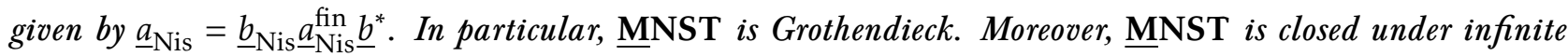

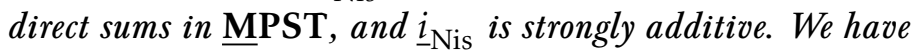

$$
\underline{b}_{\mathrm{Nis}} \underline{a}_{\mathrm{Nis}}^{\mathrm{fin}}=\underline{a}_{\mathrm{Nis}} \underline{b}_{!}, \quad \underline{a}_{s, \mathrm{Nis}} \underline{c}^{*}=\underline{c}^{\mathrm{Nis}} \underline{a}_{\mathrm{Nis}},
$$

where $\underline{c}^{\mathrm{Nis}}$ is the functor determined by Lemma-Definition 2.2.5. This functor is exact, strongly additive and has a left adjoint $\underline{c}_{\mathrm{Nis}}=\underline{a}_{\mathrm{Nis}} \underline{\underline{c}} \underline{i}_{s, \mathrm{Nis}}$.

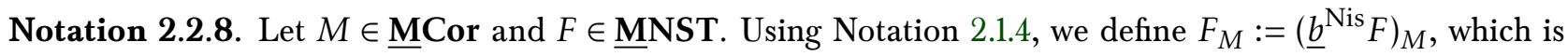
a sheaf on $(\bar{M})_{\text {Nis }}$.

Proposition 2.2.9 ([KMSY21, Proposition 4.6.3]). Let $F \in \underline{\mathrm{MNST}}$, and let $M \in \underline{\mathrm{MCor}}$. Then there is a canonical isomorphism for any $i \geq 0$ :

$$
\operatorname{Ext}_{\underline{\mathbf{M N S T}}}^{i}\left(\mathbb{Z}_{\mathrm{tr}}(M), F\right) \simeq \underset{N \in{\underline{\underline{\Sigma^{\mathrm{fin}}}}}_{\lim }}{\lim } H_{\mathrm{Nis}}^{i}\left(\bar{N}, F_{N}\right) .
$$

Moreover, we have

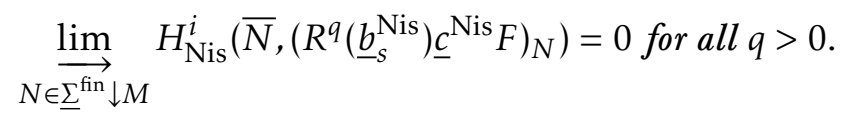

Corollary 2.2.10. We have $\operatorname{Ext}_{\underline{\mathrm{MNST}}}^{i}\left(\mathbb{Z}_{\mathrm{tr}}(M), F\right)=0$ for $i>\operatorname{dim} \bar{M}$.

Proof. For any $N \in \underline{\Sigma}^{\text {fin }} \downarrow M$, we have $\operatorname{dim} \bar{N}=\operatorname{dim} \bar{M}$. Therefore the statement follows from Proposition 2.2.9 and the known bound for Nisnevich cohomological dimension.

\section{A cd-structure on MSm}

In this section we introduce a cd-structure on MSm and describe its main properties, following the works of Miyazaki [Miy20] and Kahn-Miyazaki [KM20]. For this we need to start with the "off-diagonal" functor.

\subsection{Off-diagonal}

Definition 3.1.1. Define MEt as the category such that

(1) objects are those morphisms $f: M \rightarrow N$ in MSm such that $f^{\circ}: M^{\mathrm{o}} \rightarrow N^{\mathrm{o}}$ is étale, and

(2) a morphism from $f: M_{1} \rightarrow N_{1}$ to $g: M_{2} \rightarrow N_{2}$ is a pair of morphisms $\left(s: M_{1} \rightarrow M_{2}, t: N_{1} \rightarrow N_{2}\right)$ in MSm which commute with $f, g$ and such that $s^{\mathrm{o}}$ and $t^{\mathrm{o}}$ are open immersions.

For modulus pairs $M$ and $N$, we define the disjoint union of $M$ and $N$ by

$$
M \sqcup N:=\left(\bar{M} \sqcup \bar{N}, M^{\infty} \sqcup N^{\infty}\right) .
$$

Obviously, we have $(M \sqcup N)^{\mathrm{o}}=M^{\mathrm{o}} \sqcup N^{\mathrm{o}}$.

Theorem 3.1.2 ([Miy20, Theorem 3.1.3]). There exists a functor

$$
\text { OD }: \text { MEt } \rightarrow \text { MSm }
$$

such that for any $f: M \rightarrow N$, one has a functorial decomposition

$$
M \times{ }_{N} M \cong M \sqcup \mathrm{OD}(f) .
$$

Moreover, we have $\operatorname{OD}(f)^{\mathrm{o}}=M^{\mathrm{o}} \times_{N^{\mathrm{o}}} M^{\mathrm{o}} \backslash \Delta\left(M^{\mathrm{o}}\right)$, where $\Delta: M^{\mathrm{o}} \rightarrow M^{\mathrm{o}} \times_{N^{\circ}} M^{\mathrm{o}}$ is the diagonal morphism. In particular, if $f^{\circ}$ is an open immersion, then $\mathrm{OD}(f)^{\mathrm{O}}=\emptyset$, hence $\mathrm{OD}(f)=\emptyset$. We call the functors the off-diagonal functors. 


\subsection{The MV cd-structure}

Definition 3.2.1. Let $T$ be an object of $\mathrm{MSm}^{\mathrm{Sq}}$ of the form

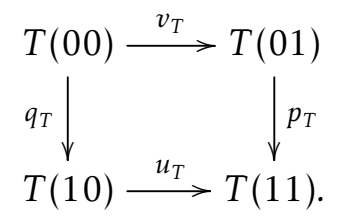

Then $T$ is called an MV-square if the following conditions hold:

(1) $T$ is a pull-back square in MSm.

(2) There exist an $\underline{M V}$-square $S(c f$. Definition 2.2.1) such that $S(11) \in \mathbf{M S m}$, and a morphism $S \rightarrow T$ in MSm $^{\text {Sq }}$ such that the induced morphism $S^{o} \rightarrow T^{\mathrm{o}}$ is an isomorphism in $\mathbf{S m}^{\mathbf{S q}}$ and $S(11) \rightarrow T(11)$ is an isomorphism in MSm. In particular, $T^{\mathrm{o}}$ is an elementary Nisnevich square.

(3) $\mathrm{OD}\left(q_{T}\right) \rightarrow \mathrm{OD}\left(p_{T}\right)$ is an isomorphism in $\mathbf{M S m}$.

We let $P_{\mathrm{MV}}$ be the cd-structure on MSm consisting of MV-squares.

The following are the main results of [Miy20].

Theorem 3.2.2 ([Miy20, Theorem 4.3.1, 4.4.1 and 4.5.1]). The cd-structure $P_{\mathrm{MV}}$ is complete and regular. The associated topology is subcanonical.

Corollary 3.2.3. Define MNS $\subset$ MPS as the full subcategory consisting of sheaves with respect to the Grothendieck topology on MSm associated with $P_{\mathrm{MV}}$. Then there exists a pair of adjoint functors

$$
\text { MPS } \underset{i_{s, \text { Nis }}}{\stackrel{a_{s, \text { Nis }}}{\longrightarrow}} \text { MNS, }
$$

where $i_{s, \mathrm{Nis}}$ is the natural inclusion and its left adjoint $a_{s, \mathrm{Nis}}$ is exact. Moreover, MNS is Grothendieck. For $F \in$ MPS, the following conditions are equivalent.

(1) $F \in$ MNS.

(2) For any MV-square $Q$ as (3.2.1), the associated sequence

$$
0 \rightarrow F(T(00)) \rightarrow F(T(10)) \times F(T(01)) \rightarrow F(T(11))
$$

is exact.

Proof. Same as for Lemma 2.2.4: the first two assertions are general facts on Grothendieck topologies, and the equivalence (i) $\Longleftrightarrow$ (ii) follows from [Voe10a, Corollary 2.17] in view of Theorem 3.2.2 (1).

The following are the main results of [KM20]. To state them, we need a definition.

\section{Definition 3.2.4.}

(1) For any square $S \in \underline{\mathbf{M S m}}{ }^{\mathrm{Sq}}$, we define categories $\operatorname{Comp}(S)$ as the full subcategories of $S \downarrow \mathbf{M S m}^{\text {Sq }}$ consisting of those objects $S \rightarrow T$ such that $S(i j) \rightarrow T(i j)$ belongs to $\operatorname{Comp}(S(i j))$ for any $(i j) \in \mathbf{S q}$. Here, $\operatorname{Comp}(-)$ is from Definition 1.2.2.

(2) For an $\underline{\mathrm{MV}}^{\text {fin }}$-square $S$ in $\underline{\mathbf{M S}} \mathbf{m}^{\text {fin }}$, an object $S \rightarrow \tau^{\mathbf{S q}}(Q)$ in $\operatorname{Comp}(S)$ is an MV-completion of $S$ if $Q$ is an MV-square. We write

$$
\operatorname{Comp}(S)^{\mathrm{MV}} \subset \operatorname{Comp}(S)
$$

for the full subcategory consisting of MV-completions of $S$.

Theorem 3.2.5. The following assertions hold. 
(1) [KM20, Theorem 1]. The functor $\tau_{s}: \mathbf{M S m} \rightarrow \underline{\text { MSm }}$ is continuous in the sense of [SGA4, exposé III] for the topologies given by $P_{\mathrm{MV}}$ and $P_{\mathrm{MV}}$.

(2) [KM20, Theorem 1.5.6]. For any $\underline{\mathrm{MV}}^{\text {fin }}$-square $S$ such that $\bar{S}(11)$ is normal, $\operatorname{Comp}(S)^{\mathrm{MV}}$ is cofinal in $\operatorname{Comp}(S)$.

Corollary 3.2.6. Let $S$ be an $\underline{\mathrm{MV}}^{\text {fin }}$-square such that $\bar{S}(11)$ is normal. Then, for any $i, j \in\{0,1\}$, the subcategory of $\operatorname{Comp}(S(i j))$ defined by

$$
\left\{T(i j) \mid T \in \operatorname{Comp}(S)^{\mathrm{MV}}\right\}
$$

is cofinal in $\operatorname{Comp}(S(i j))$.

Proof. By Theorem 3.2.5(1), it suffices to prove that the subcateogry $\{T(i j) \mid T \in \operatorname{Comp}(S)\}$ is cofinal in $\operatorname{Comp}(S(i j))$ for any $i, j \in\{0,1\}$. To show this we need the following

Lemma 3.2.7. For any morphism $f: V \rightarrow U$ in $\underline{M S m}^{\text {fin }}$ and for any $M \in \operatorname{Comp}(U)$, there exists $N \in$ $\operatorname{Comp}(V)$ such that $f$ induces a morphism $N \rightarrow M$ in $\mathbf{M S m}^{\text {fin }}$.

Proof. Take any $N \in \operatorname{Comp}(V)$. Let $\Gamma$ be the graph of the rational map $\bar{N} \rightarrow \bar{M}$ and let $\pi: \Gamma \rightarrow \bar{N}$. Then $\pi$ induces an isomorphism $N^{\prime}:=\left(\Gamma, \pi^{*} N^{\infty}\right) \cong N$ and $N^{\prime} \in \operatorname{Comp}(V)$. Therefore, by replacing $N$ with $N^{\prime}$, we may assume that $\bar{f}: \bar{U} \rightarrow \bar{V}$ extends to a morphism of schemes $\bar{p}: \bar{N} \rightarrow \bar{M}$. Moreover, by taking blow up $\mathbf{B} \mathbf{l}_{\bar{N}-\bar{V}}(\bar{N}) \rightarrow \bar{N}$ and pulling back the divisor, we may assume that there exists an effective Cartier divisor $D$ on $\bar{N}$ with $\bar{N}-\bar{V}=|D|$. Since the admissiblity of $V \rightarrow U$ implies $\left.\bar{p}^{*} M^{\infty}\right|_{\bar{V}^{N}}=\left.\bar{f}^{*} U^{\infty}\right|_{\bar{V}^{N}} \leq\left. V^{\infty}\right|_{\bar{V}^{N}}=\left.N^{\infty}\right|_{\bar{V}^{N}}$, [Miy19, Lemma 3.14] shows that there exists a positive integer $n$ such that $\left.\bar{p}^{*} M^{\infty}\right|_{\bar{N}^{N}} \leq\left.\left(N^{\infty}+n D\right)\right|_{\bar{N}^{N}}$. Then $N^{\prime \prime}:=\left(\bar{N}, N^{\infty}+n D\right) \in \operatorname{Comp}(V)$, it dominates $N$ and $\bar{p}$ induces a morphism $N^{\prime \prime} \rightarrow M$ in $\underline{\mathbf{M S m}}{ }^{\text {fin }}$, as desired.

The corollary immediately follows from the lemma when $(i, j)=(1,1)$. We prove the case $(i, j)=(1,0)$. Take any $N \in \operatorname{Comp}(S(10))$ and any $T \in \operatorname{Comp}(S)$. Since $\operatorname{Comp}(S(10))$ is filtered, there exists $T^{\prime}(10) \in$ $\operatorname{Comp}(S(10))$ which dominates both $N$ and $T(10)$. By the lemma there exists $T^{\prime}(00) \in \operatorname{Comp}(S(00))$ such that $S(00) \rightarrow S(10)$ extends to $T^{\prime}(00) \rightarrow T(10)$. Since $\operatorname{Comp}(S(00))$ is ordered we may assume that $T^{\prime}(00)$ dominates $T(00)$. Then the resulting diagram

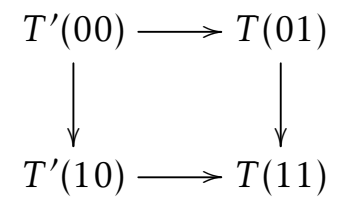

is an object of $\operatorname{Comp}(S)$ dominating $T$, and $T^{\prime}(10)$ dominates $N$ by construction. This proves the case $(i, j)=(1,0)$. The proof for $(i, j)=(0,1)$ is completely the same.

Finally we prove the case $(i, j)=(0,0)$. Take any $N \in \operatorname{Comp}(S(00))$ and take any $T \in \operatorname{Comp}(S)$. Since $\operatorname{Comp}(S(00))$ is filtered, there exists $T^{\prime}(00) \in \operatorname{Comp}(S(00))$ which dominates both $N$ and $T(00)$. Then the square obtained by replacing $T(00)$ with $T^{\prime}(00)$ dominates $T$. This finishes the proof of Corollary 3.2.6.

Remark 3.2.8. The essential point of the above proof is the fact that the diagram category Sq does not have a loop, and therefore the use of the graph trick terminates in finitely many steps. We remark that we can generalize the proof to a much more abstract argument, $c f$. [KM18, Lemma C.6].

\section{Sheaves on MSm and MCor}

\subsection{Sheaves on MSm}

Lemma 4.1.1. The category MNS is closed under infinite direct sums and the inclusion functor $i_{s, \mathrm{Nis}}$ is strongly additive. 
Proof. Indeed, the sheaf condition is tested on finite diagrams, hence the presheaf given by a direct sum of sheaves is a sheaf.

Theorem 4.1.2. The following assertions hold.

(1) We have $\tau_{s}^{*}(\underline{\mathrm{MNS}}) \subset \mathrm{MNS}$ and $\tau_{s !}(\mathrm{MNS}) \subset \underline{\mathrm{MNS}}$, where $\tau_{s}^{*}$ and $\tau_{s !}$ are from Lemma 1.2.3. Hence we obtain functors

such that

$$
\tau_{s}^{\mathrm{Nis}}: \underline{\mathrm{MNS}} \rightarrow \text { MNS } \quad \text { and } \quad \tau_{s, \mathrm{Nis}}: \text { MNS } \rightarrow \underline{\mathrm{MNS}}
$$

$$
i_{s, \mathrm{Nis}} \tau_{s}^{\mathrm{Nis}}=\tau_{s}^{*} \underline{i}_{s, \mathrm{Nis}}, \quad \tau_{s !} i_{s, \mathrm{Nis}}=\underline{i}_{s, \mathrm{Nis}} \tau_{s, \mathrm{Nis}}
$$

and that $\tau_{s}^{\mathrm{Nis}}$ is right adjoint to $\tau_{s, \mathrm{Nis}}$. (See (1.1.1) for $\tau_{s}$.)

(2) For $F \in$ MPS, one has $F \in$ MNS if and only if $\tau_{s, !} F \in \underline{\text { MNS. }}$

(3) We have

$$
\underline{a}_{s, \mathrm{Nis}} \tau_{s !}=\tau_{s, \mathrm{Nis}} a_{s, \mathrm{Nis}},
$$

(4) The functor $\tau_{s, \text { Nis }}$ is fully faithful and exact. Moreover, the functor $\tau_{s}^{\mathrm{Nis}}$ preserves injectives.

Proof. First we prove (1). The first assertion follows from the continuity of $\tau_{s}$ (Theorem 3.2.5 (1)). Similarly, the second assertion morally follows from the "continuity of $\tau_{s}^{!}$" (see [KM20, Remark 1]): we give a proof based on Theorem 3.2.5 (2).

Take $F \in$ MNS. By Lemma 2.2.4, it suffices to show that the sequence

$$
0 \rightarrow \tau_{!} F(M) \rightarrow \tau_{!} F(U) \times \tau_{!} F(V) \rightarrow \tau_{!} F(W)
$$

is exact for any $Q \in P_{\underline{\mathrm{MV}}}$ as in (2.2.1). By Remark 1.1.1 (1), we may assume that $\bar{M}$ is normal. Since a filtered colimit of exact sequences of abelian groups is exact, the desired assertion follows from Lemma 1.2.3 (4), Corollary 3.2.3 and Corollary 3.2.6. Finally the adjointness follows from Lemma 1.2.3 (2). This completes the proof of (1).

(2) follows from (1) and Id $\cong \tau_{s}^{*} \tau_{s, !}$ by Lemma 1.2 .3 (2).

(3) follows from (4.1.1) by adjunction.

In (4), the exactness of $\tau_{s, \mathrm{Nis}}$ follows from Lemma 1.2.6 (4) applied to $c=\tau_{s, !}$, using (1), (3) and Lemma 2.2.4. It then implies the preservation of injectives by $\tau_{s}^{\text {Nis }}$. The full faithfulness of $\tau_{s, \text { Nis }}$ follows from that of $\tau_{s !}$ (Lemma 1.2.3 (2)) and (4.1.1).

Remark 4.1.3. By [SGA4, exposé II, Proposition 1.3], the continuity of $\tau_{s}$ implies a priori the existence of a left adjoint $\tau_{s, \mathrm{Nis}}$ to $\tau_{s}^{\mathrm{Nis}}$ on sheaves of sets, having the following properties:

(1) (4.1.2);

(2) $\tau_{s, \mathrm{Nis}}=a_{s, \mathrm{Nis}} \tau_{s, !} i_{s, \mathrm{Nis}}$;

(3) $\tau_{s, \text { Nis }}$ commutes with representable colimits;

(4) $\tau_{s, \mathrm{Nis}}$ sends a representable sheaf to the corresponding representable sheaf.

All this extends to sheaves of abelian groups by [SGA4, exposé II, Proposition 6.3.1]. The one property which is missing is the right formula of (4.1.1). From (4.1.2), we deduce a base change morphism

$$
\tau_{s !} i_{s, \mathrm{Nis}} \Rightarrow \underline{i}_{s, \mathrm{Nis}} \tau_{s, \mathrm{Nis}}
$$

that we have shown above to be an isomorphism. By (4), this isomorphism is clear on the generators $\mathbb{Z}[M]$

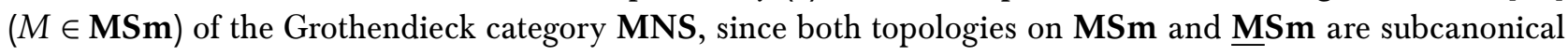
(Theorem 2.2.2 and 3.2.2 (1)). But both sides of (4.1.3) are a composition of left and right exact functors, so this is not sufficient to get the general case. Thus the recourse to Theorem 3.2.5 (2) seems necessary to prove Theorem 4.1.2 (1). 


\subsection{Sheaves on MCor}

The following is an analogue of Lemma-Definition 2.2.5:

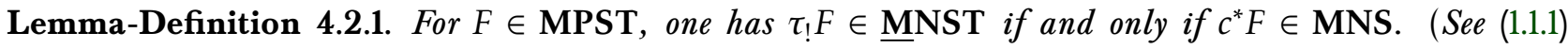
for c.) We write MNST for the full subcategory of MPST consisting of those $F \in$ MPST that enjoy these

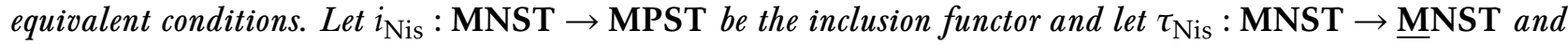
$c^{\mathrm{Nis}}: \mathrm{MNST} \rightarrow \mathrm{MNS}$ be such that

$$
\underline{i}_{\mathrm{Nis}} \tau_{\mathrm{Nis}}=\tau_{!} i_{\mathrm{Nis}}, \quad i_{s, \mathrm{Nis}} c^{\mathrm{Nis}}=c^{*} i_{\mathrm{Nis}} .
$$

Proof. Let $F \in$ MPST. Then

$$
\tau_{!} F \in \underline{\mathbf{M N S T}} \Rightarrow \tau_{s !} c^{*} F=\underline{c}^{*} \tau_{!} F \in c^{*} \underline{\mathbf{M N S T}} \subset \underline{\mathbf{M N S}}
$$

where we used (1.2.3) and Lemma-Definition 2.2.5; by Theorem 4.1.2 (2), this implies $c^{*} F \in$ MNS. This reasoning can be reversed.

\section{Lemma 4.2.2.}

(1) For any $M \in$ MCor, the presheaf $\mathbb{Z}_{\mathrm{tr}}(M) \in$ MPST belongs to MNST.

(2) We have a natural isomorphism

$$
\tau_{s, \mathrm{Nis}} c^{\mathrm{Nis}} \cong \underline{c}^{\mathrm{Nis}} \tau_{\mathrm{Nis}}
$$

Proof. (1) follows from Theorem 2.2.7, since $\tau_{!} \mathbb{Z}_{\mathrm{tr}}(M)=\mathbb{Z}_{\mathrm{tr}}(\tau M)$. (2) is a consequence of (4.2.1) and the corresponding isomorphism for presheaves (1.2.3).

Lemma 4.2.3. Let us consider the "2-Cartesian product category" $\mathrm{MNS} \times_{\mathrm{MNS}} \underline{\mathrm{MNST}}$, that is, the category of

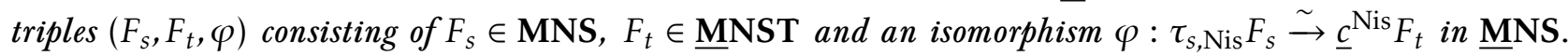
The functor

$$
\mathrm{MNST} \rightarrow \text { MNS } \times \underline{\text { MNS }} \underline{\text { MNST, }}
$$

defined by $F \mapsto\left(c^{\mathrm{Nis}} F, \tau_{\mathrm{Nis}} F, \theta_{F}\right)$, where $\theta_{F}: \tau_{s, \mathrm{Nis}} c^{\mathrm{Nis}} F \stackrel{\sim}{\rightarrow} \underline{c}^{\mathrm{Nis}} \tau_{\mathrm{Nis}} F$ is from (4.2.2), is an equivalence of categories.

Proof. The same statement was proven for presheaves in [KMSY21, Lemma 2.7.1]; full faithfulness follows from this, and essential surjectivity follows from Lemma-Definition 4.2.1.

Theorem 4.2.4. The following assertions hold.

(1) The functor $i_{\mathrm{Nis}}$ is strongly additive and has an exact left adjoint $a_{\mathrm{Nis}}$. Consequently, MNST is Grothendieck. We have

$$
c^{\mathrm{Nis}} a_{\mathrm{Nis}}=a_{s, \mathrm{Nis}} c^{*}, \quad \tau_{\mathrm{Nis}} a_{\mathrm{Nis}}=\underline{a}_{\mathrm{Nis}} \tau_{!}
$$

(2) The functor $c^{\mathrm{Nis}}$ has a left adjoint $c_{\mathrm{Nis}}=a_{\mathrm{Nis}} c_{!} i_{\mathrm{Nis}}$. Moreover, $c^{\mathrm{Nis}}$ is exact, strongly additive, and faithful.

Proof. By Definition of MNST, the strong addtivity of $i_{\text {Nis }}$ follows from that of $\underline{i}_{\text {Nis }}$ (Theorem 2.2 .7 and Lemmma 1.2.6 (2)). We then use Lemma 4.2.3 and [KMSY21, Lemma 2.7.1] to construct $a_{\mathrm{Nis}}$ by patching $a_{s, \mathrm{Nis}}$ and $\underline{a}_{\mathrm{Nis}}$ over $\underline{a}_{s, \mathrm{Nis}}$, i.e. we want $a_{\mathrm{Nis}}$ to verify (4.2.3); a formal argument shows that such a patching 
is determined by the second isomorphism of (2.2.3) and by the one of (4.1.2).

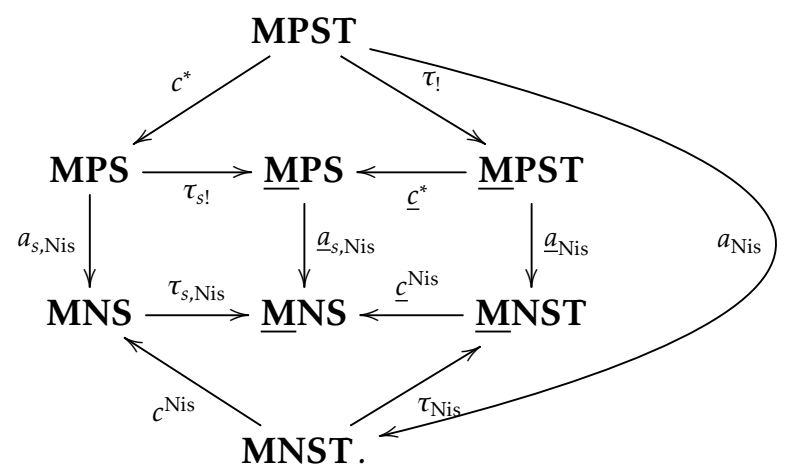

The second isomorphism of (4.2.3) easily implies that $a_{\mathrm{Nis}}$ is left adjoint to $i_{\mathrm{Nis}}$. Then (2) follows from Lemma 1.2.5, Lemma 4.1.1 and Lemma 1.2.6 (3).

Finally, the exactness of $a_{\mathrm{Nis}}$ is a consequence of the first isomorphism of (4.2.3) since $c^{\text {Nis }}$ is faithfully exact as we have just shown.

Lemma 4.2.5. The following assertions hold.

(1) We have $\tau^{*}(\underline{\text { MNST }}) \subset$ MNST.

(2) Let $\tau^{\mathrm{Nis}}: \underline{\mathrm{MNST}} \rightarrow \mathrm{MNST}$ be the functor characterized by

$$
\tau^{*} \underline{\mathrm{N}}_{\mathrm{Nis}}=i_{\mathrm{Nis}} \tau^{\mathrm{Nis}}
$$

Then $\tau^{\mathrm{Nis}}$ is a right adjoint of $\tau_{\mathrm{Nis}}$, and $\tau_{\mathrm{Nis}}$ is fully faithful, exact, and strongly additive. Moreover, $\tau^{\mathrm{Nis}}$ preserves injectives and is strongly additive.

Remark 4.2.6. We will see in Theorem 5.1 .1 below that $\tau^{\mathrm{Nis}}$ is also exact.

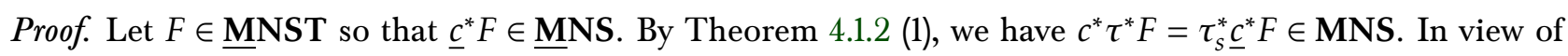
Lemma-Definition 4.2.1, this proves that $\tau^{*} F \in$ MNST, whence (1).

In (2), the existence (and uniqueness) of $\tau^{\text {Nis }}$ follows from (1) (and the full faithfulness of $\underline{i}_{\mathrm{Nis}}$ and $i_{\mathrm{Nis}}$ ). The adjointness is shown by using the full faithfulness of $\underline{i}_{\mathrm{Nis}}$ and $i_{\mathrm{Nis}}$, the adjoint pair $\left(\tau_{1}, \tau^{*}\right),(4.2 .1)$ and (4.2.4). Similarly, the full faithfulness of $\tau_{\mathrm{Nis}}$ follows from that of $\tau_{!}$(Proposition 1.2.3) and (4.2.1). The strong additivity (resp. exactness) of $\tau_{\text {Nis }}$ follows from Proposition 1.2.3, Theorem 4.2.4 and Lemma 1.2.6 (2) (resp. (4)), applied with $c=\tau_{!}$; the latter implies that $\tau^{\text {Nis }}$ preserves injectives. Finally, its strong additivity is reduced to that of $\tau^{*}$ (Lemma 1.2.3 (1)), $\underline{i}_{\text {Nis }}$ (Theorem 2.2.7) and $i_{\text {Nis }}$ (Theorem 4.2.4) by the full faithfulness of $i_{\text {Nis }}$.

\section{Cohomology in MNST}

\subsection{Main result}

We begin with the following.

Theorem 5.1.1. The functor $\tau^{\mathrm{Nis}}: \underline{\mathrm{MNST}} \rightarrow$ MNST from Lemma 4.2 .5 is exact.

The proof will be given later in this section (see Corollary 5.5.1). We now deduce its consequences.

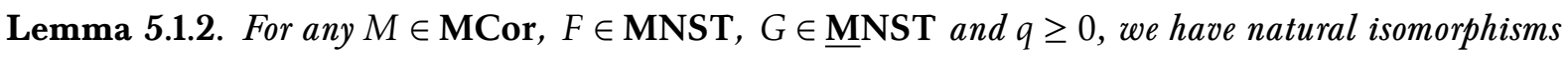

$$
\begin{aligned}
& \operatorname{Ext}_{\text {MNST }}^{q}\left(\mathbb{Z}_{\mathrm{tr}}(M), \tau^{\mathrm{Nis}} G\right) \cong \operatorname{Ext}_{\underline{\mathbf{M N S T}}}^{q}\left(\mathbb{Z}_{\mathrm{tr}}(M), G\right), \\
& \operatorname{Ext}_{\mathbf{M N S T}}^{q}\left(\mathbb{Z}_{\mathrm{tr}}(M), F\right) \cong \operatorname{Ext}_{\underline{\mathbf{M N S T}}}^{q}\left(\mathbb{Z}_{\mathrm{tr}}(M), \tau_{\mathrm{Nis}} F\right) .
\end{aligned}
$$


Proof. By Theorem 5.1.1, $\tau^{\mathrm{Nis}}$ is exact and it preserves injectives by Lemma 4.2.5. Hence we have

$$
R^{q}\left(i_{\mathrm{Nis}}\right) \tau^{\mathrm{Nis}} G=R^{q}\left(i_{\mathrm{Nis}} \tau^{\mathrm{Nis}}\right) G=R^{q}\left(\tau^{*} \underline{i}_{\mathrm{Nis}}\right) G=\tau^{*} R^{q} \underline{\underline{N}}_{\mathrm{Nis}} G
$$

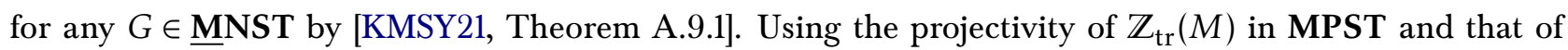
$\tau_{!} \mathbb{Z}_{\mathrm{tr}}(M)=\overline{\mathbb{Z}}_{\mathrm{tr}}(M)$ in $\underline{\text { MPST}}$, and using Proposition A.1.1 twice, we get isomorphisms

$$
\begin{aligned}
\operatorname{Ext}_{\mathrm{MNST}}^{q}\left(a_{\mathrm{Nis}} \mathbb{Z}_{\mathrm{tr}}(M), \tau^{\mathrm{Nis}} G\right) \simeq \operatorname{MPST}\left(\mathbb{Z}_{\mathrm{tr}}(M), R^{q}\left(i_{\mathrm{Nis}}\right) \tau^{\mathrm{Nis}} G\right) \\
\simeq \operatorname{MPST}\left(\mathbb{Z}_{\mathrm{tr}}(M), \tau^{*} R^{q} \underline{\underline{i}}_{\mathrm{Nis}} G\right) \simeq \underline{\operatorname{MPST}}\left(\tau_{!} \mathbb{Z}_{\mathrm{tr}}(M), R^{q} \underline{\underline{i}}_{\mathrm{Nis}} G\right) \\
\left.\simeq \underline{\operatorname{MPST}}\left(\mathbb{Z}_{\mathrm{tr}}(M), R^{q} \underline{\underline{i}}_{\mathrm{Nis}} G\right) \simeq \operatorname{Ext}_{\underline{\mathrm{MNST}}}^{q} \underline{a}_{\mathrm{Nis}} \mathbb{Z}_{\mathrm{tr}}(M), G\right) .
\end{aligned}
$$

Moreover, $a_{\mathrm{Nis}} \mathbb{Z}_{\mathrm{tr}}(M)=\mathbb{Z}_{\mathrm{tr}}(M)$ and $\underline{a}_{\mathrm{Nis}} \mathbb{Z}_{\mathrm{tr}}(M)=\mathbb{Z}_{\mathrm{tr}}(M)$ by Theorem 2.2.7 and Lemma 4.2.2 (1), whence the first formula by evaluating both sides at $M$. The second one follows from the first by taking $G=\tau_{\mathrm{Nis}} F$, since $\tau^{\mathrm{Nis}} \tau_{\mathrm{Nis}}=\mathrm{Id}$.

Let $M \in$ MCor and $F \in$ MNST. Using Notation 2.2.8, we define $F_{M}:=\left(\tau_{\mathrm{Nis}} F\right)_{M}$, which is a sheaf on $(\bar{M})_{\text {Nis }}$.

Theorem 5.1.3. For any $p \geq 0, M \in$ MCor and $F \in$ MNST, we have a natural isomorphism

$$
\operatorname{Ext}_{\text {MNST }}^{p}\left(\mathbb{Z}_{\text {tr }}(M), F\right) \simeq \underset{N \in \sum^{\mathrm{fin}} \downarrow M}{\lim _{\mathrm{Nis}}} H_{N}^{p}\left(\bar{N}, F_{N}\right) .
$$

Moreover, we have

$$
\underset{N \in \underline{\Sigma}_{\sum^{\mathrm{fin}}} \downarrow M}{\lim } H_{\mathrm{Nis}}^{p}\left(\bar{N},\left(R^{q}\left(\underline{b}_{s}^{\mathrm{Nis}}\right) \underline{c}^{\mathrm{Nis}} \tau_{\mathrm{Nis}} F\right)_{N}\right)=0 \text { for all } q>0 .
$$

Proof. Combine Proposition 2.2.9, Lemma 4.2.5 and Lemma 5.1.2.

Corollary 5.1.4. We have $\operatorname{Ext}_{\mathrm{MNST}}^{q}\left(\mathbb{Z}_{\mathrm{tr}}(M), F\right)=0$ for $q>\operatorname{dim} \bar{M}$.

Proof. Same as for Corollary 2.2.10.

\subsection{A generation lemma}

We now start proving Theorem 5.1.1. We need some preliminaries.

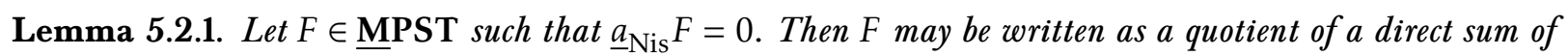

$$
\mathbb{Z}_{\mathrm{tr}}(M / U):=\operatorname{Coker}\left(\mathbb{Z}_{\mathrm{tr}}(U) \rightarrow \mathbb{Z}_{\mathrm{tr}}(M)\right),
$$

where $M \in \underline{\mathrm{MS}}, U \rightarrow M$ is a covering for the Grothendieck topology on $\underline{\mathrm{MSm}}^{\text {fin }}$ associated to $P_{\underline{\mathrm{MV}}^{\text {fin }}}$ from

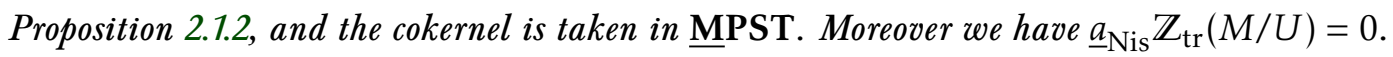

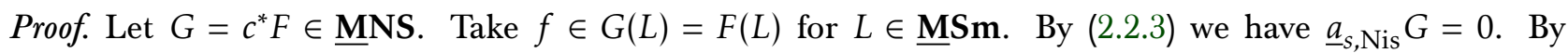
[KMSY21, Lemma 4.3.2], we have $\varphi^{*} f=0$ for a cover $\varphi: U \rightarrow M \rightarrow L$ in $\underline{\mathbf{M S m}}_{\mathrm{Nis}}$, where $U \rightarrow M$ is a

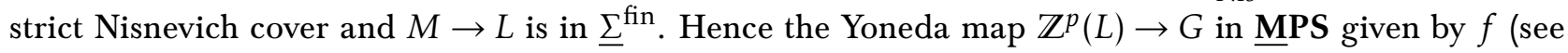
Definition 1.2.1 (4) for $\left.\mathbb{Z}^{p}(L)\right)$ factors through

$$
\mathbb{Z}^{p}(L / U):=\operatorname{Coker}\left(\mathbb{Z}^{p}(U) \rightarrow \mathbb{Z}^{p}(L)\right)
$$

(see Definition 1.2.1 (4) for $\left.\mathbb{Z}^{p}(-)\right)$. By the adjunction $\left(c_{!}, c^{*}\right)$ this induces a map $c_{!} \mathbb{Z}^{p}(L / U) \rightarrow F$, and

$$
c_{!} \mathbb{Z}^{p}(L / U) \simeq \operatorname{Coker}\left(c_{!} \mathbb{Z}^{p}(U) \rightarrow c_{!} \mathbb{Z}^{p}(L)\right) \simeq \operatorname{Coker}\left(\mathbb{Z}_{\mathrm{tr}}(U) \rightarrow \mathbb{Z}_{\mathrm{tr}}(L)\right)
$$

where the first isomorphism follows from the right exactness of $c_{!}$as a left adjoint. This implies that the

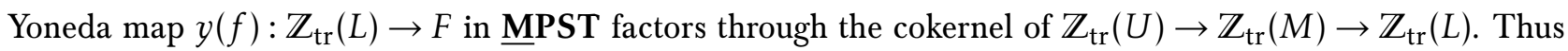
we get an induced map $\overline{y(f)}: \mathbb{Z}_{\mathrm{tr}}(M / U) \rightarrow F$. Since the map $\mathbb{Z}_{\mathrm{tr}}(M) \rightarrow \mathbb{Z}_{\mathrm{tr}}(L)$ is an isomorphism in MPST, the image of $y(f)$ coincides with that of $\overline{y(f)}$. Collecting them over all pairs $(L, f)$, this proves the first part of the lemma. Finally the last statement follows from [KMSY21, Theorem 4.5.7]. 


\subsection{The $\tau$ construction}

Definition 5.3.1. Let $M, N \in \underline{\text { MCor. }}$

(1) We put

$$
\mathbb{Z}_{\mathrm{tr}}(M)^{\tau}=\tau_{!} \tau^{*} \mathbb{Z}_{\mathrm{tr}}(M) \in \underline{\text { MPST }} .
$$

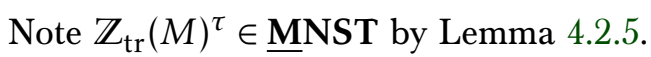

(2) Let $\underline{\operatorname{MCor}}{ }^{\tau}(N, M)$ be the subgroup of $\underline{\operatorname{MCor}}(N, M)$ generated by elementary correspondences $Z$ in $\operatorname{Cor}\left(N^{\circ}, M^{\circ}\right)$ which satisfy the condition:

(ه) There exists a dense open immersion $j: \bar{N} \hookrightarrow \bar{L}$ with $\bar{L}$ proper such that the closure $\bar{Z}$ of $Z$ in $\bar{L} \times \bar{M}$ is proper over $\bar{L}$.

Lemma 5.3.2. For $N, M$ as above, the condition ( $\bullet$ is independent of the choice of $j: \bar{N} \hookrightarrow \bar{L}$, and we have

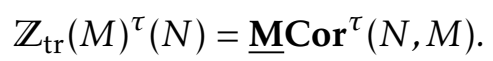

Proof. If $j^{\prime}: \bar{N} \hookrightarrow \bar{L}^{\prime}$ is another choice equipped with (proper) surjective $f: \bar{L} \rightarrow \bar{L}^{\prime}$ such that $j^{\prime}=f j$, writing $\bar{Z}^{\prime} \subset \bar{L}^{\prime} \times \bar{M}$ for the closure of $Z, f$ induces a proper surjective map $\bar{Z}^{\prime} \rightarrow \bar{Z}$. Then it is easy to see that $\bar{Z}^{\prime}$ is proper over $\bar{L}^{\prime}$ if and only if so is $\bar{Z}$ over $\bar{L}$. This proves the first assertion. To show the second assertion, we note that by Lemma 1.2.3 (3),

$$
\mathbb{Z}_{\mathrm{tr}}(M)^{\tau}(N)=\underset{L \in \operatorname{Comp}(N)}{\lim _{\longrightarrow}} \underline{\operatorname{MCor}}(L, M) .
$$

The second assertion follows from this using the first assertion (see the proof of [KMSY21, Lemma 1.8.3]).

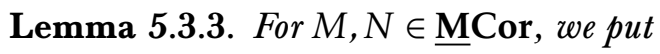

$$
\mathbb{Z}_{\operatorname{tr}}^{\mathrm{fin}}(M)^{\tau}(N)=\underline{\operatorname{MCor}}^{\mathrm{fin}}(N, M) \cap \underline{\operatorname{M}} \operatorname{Cor}^{\tau}(N, M) \subset \underline{\operatorname{MC}} \operatorname{Cor}(N, M) .
$$

Then $\mathbb{Z}_{\mathrm{tr}}^{\mathrm{fin}}(M)^{\tau}$ defines an object of $\underline{\mathrm{MPST}}^{\mathrm{fin}}$. Moreover we have

$$
\underline{b}_{!} \mathbb{Z}_{\mathrm{tr}}^{\mathrm{fin}}(M)^{\tau}=\mathbb{Z}_{\mathrm{tr}}(M)^{\tau}
$$

Proof. The first assertion follows from Lemma 5.3.2. For $N^{\prime} \in \underline{\Sigma}^{\text {fin }} \downarrow N$ (see Definition 2), Lemma 5.3.2 implies

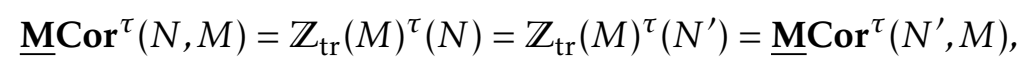

where the second equality follows from the fact that $N^{\prime} \simeq N$ in MCor. By definition this implies

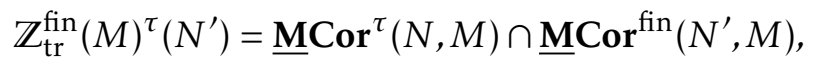

which proves the second assertion in view of the isomorphisms

$$
\begin{aligned}
& \underline{b}_{!} \mathbb{Z}_{\mathrm{tr}}^{\mathrm{fin}}(M)^{\tau}(N)=\underset{N^{\prime} \in \underline{\Sigma}^{\mathrm{fin}} \downarrow N}{\underset{\lim }{\longrightarrow}} \mathbb{Z}_{\mathrm{tr}}^{\mathrm{fin}}(M)^{\tau}\left(N^{\prime}\right), \\
& \underline{\operatorname{MCor}}(N, M)=\underset{N^{\prime} \in \underline{\Sigma}^{\mathrm{fin}} \downarrow N}{\underset{\lim }{\longrightarrow}} \underline{\operatorname{MCor}}{ }^{\mathrm{fin}}\left(N^{\prime}, M\right),
\end{aligned}
$$

which hold by (1.2.2) and [KMSY21, Proposition 1.9.2].

Remark 5.3.4. We can prove that $\mathbb{Z}_{\mathrm{tr}}^{\mathrm{fin}}(M)^{\tau}$ lies in $\underline{\mathrm{MNST}}^{\text {fin }}$ (so that we may remove $\underline{a}_{\mathrm{Nis}}^{\mathrm{fin}}$ in Theorem 5.4.1(1) below). 


\subsection{Exactness of a certain Čech complex}

Theorem 5.4.1. Let $p: U \rightarrow M$ be a covering for the Grothendieck topology on $\underline{\mathrm{MSm}}^{\text {fin }}$ associated to $P_{\underline{\mathrm{MV}}}{ }^{\text {fin }}$ from Proposition 2.1.2. Denote by $U \times_{M} U$ the fiber product in $\underline{\mathbf{M S m}}^{\text {fin }}$ (see [KMSY21, Proposition 1.10.4 and Corollary 1.10.7]).

(1) The Cech complex

$$
\cdots \rightarrow \underline{a}_{\mathrm{Nis}}^{\mathrm{fin}} \mathbb{Z}_{\mathrm{tr}}^{\mathrm{fin}}\left(U \times \times_{M} U\right)^{\tau} \rightarrow \underline{a}_{\mathrm{Nis}}^{\mathrm{fin}} \mathbb{Z}_{\mathrm{tr}}^{\mathrm{fin}}(U)^{\tau} \rightarrow \underline{a}_{\mathrm{Nis}}^{\mathrm{fin}} \mathbb{Z}_{\mathrm{tr}}^{\mathrm{fin}}(M)^{\tau} \rightarrow 0
$$

is exact in MNST $^{\text {fin }}$.

(2) The Cech complex

$$
\cdots \rightarrow \mathbb{Z}_{\mathrm{tr}}\left(U \times_{M} U\right)^{\tau} \rightarrow \mathbb{Z}_{\mathrm{tr}}(U)^{\tau} \rightarrow \mathbb{Z}_{\mathrm{tr}}(M)^{\tau} \rightarrow 0
$$

is exact in $\underline{\mathrm{MNST}}$.

Theorem 5.4.1 (2) follows from (1) by applying the exact functor $\underline{b}_{\mathrm{Nis}}$ from Proposition 2.2.9 and using isomorphisms

$$
\underline{b}_{\mathrm{Nis}} \underline{a}_{\mathrm{Nis}} \mathbb{Z}_{\mathrm{tr}}^{\mathrm{fin}}(M)^{\tau} \simeq \underline{a}_{\mathrm{Nis}} \underline{b}_{!} \mathbb{Z}_{\mathrm{tr}}^{\mathrm{fin}}(M)^{\tau} \simeq \underline{a}_{\mathrm{Nis}} \mathbb{Z}_{\mathrm{tr}}(M)^{\tau}=\mathbb{Z}_{\mathrm{tr}}(M)^{\tau},
$$

where the first isomorphism follows from (2.2.3), the second from Lemma 5.3.3 and the last equality follows

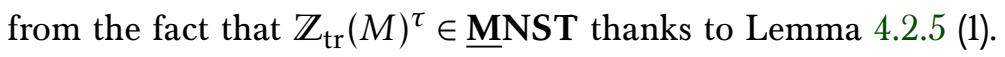

We need some preliminaries for the proof of (1). It is inspired by that of [KMSY21, Theorem 3.4.1], with

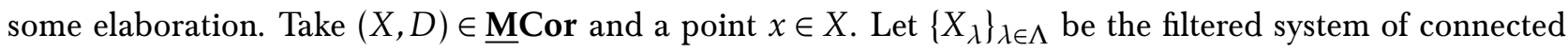
affine étale neighborhoods of $x \in X$. Let

$$
\bar{S}=\lim _{\lambda \in \Lambda} X_{\lambda}
$$

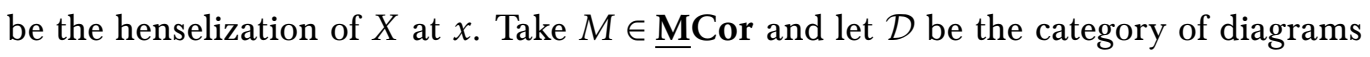

$$
\bar{S} \stackrel{f}{\leftarrow} Z \stackrel{g}{\rightarrow} \bar{M}
$$

of $k$-schemes with $f$ quasi-finite such that $Z \rightarrow \bar{S} \times \bar{M}$ is a closed immersion and $V \not \subset \bar{S} \times M^{\infty}$ for any irreducible component $V$ of $Z$. We denote (5.4.2) by $(Z, f, g)$. A morphism from $(Z, f, g)$ to $\left(Z^{\prime}, f^{\prime}, g^{\prime}\right)$ is given by a morphism $\varphi: Z \rightarrow Z^{\prime}$ which fits into a commutative diagram

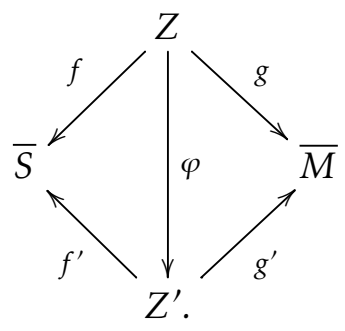

Note that $\varphi$ is automatically a closed immersion, so $\mathcal{D}$ is a cofiltered ordered set as it is stable under unions. For $(Z, f, g) \in \mathcal{D}$ let $E(Z)=E(Z, f, g)$ be the set of irreducible components $V$ of $Z$ which belong to $\underline{M C o r}^{\text {fin }}((\bar{S}, D), M)$, i.e. such that $\left.f\right|_{V}$ is finite and surjective over an irreducible component of $\bar{S}$ and satisfies the admissibility condition:

$$
\left(f \circ i_{V} \circ v\right)^{*}\left(D \times_{X} \bar{S}\right) \geq\left(g \circ i_{V} \circ v\right)^{*}\left(M^{\infty}\right),
$$

where $v: V^{N} \rightarrow V$ is the normalization and $i_{V}: V \hookrightarrow Z$ is the inclusion. Let $E^{\tau}(Z) \subset E(Z)$ be the subset of those $V$ which belong to $\mathbb{Z}_{\mathrm{tr}}^{\mathrm{fin}}(M)^{\tau}(\bar{S}, D)$, i.e. satisfying the following condition: there exists $\lambda \in \Lambda$ such that $(Z, f, g)\left(\right.$ resp. $V \hookrightarrow Z$ ) is the base change via $\bar{S} \rightarrow X_{\lambda}$ of

$$
\left.X_{\lambda} \stackrel{f_{\lambda}}{\longleftarrow} Z_{\lambda} \stackrel{g_{\lambda}}{\longrightarrow} \bar{M} \quad \text { (resp. } V_{\lambda} \hookrightarrow Z_{\lambda}\right),
$$


where $V_{\lambda}$ is an irreducible component of $Z_{\lambda}$ satisfying the condition:

(*) ${ }_{\lambda} V_{\lambda}$ is finite over $X_{\lambda}$ and satisfies the admissibility condition

$$
\left(f_{\lambda} \circ i_{V_{\lambda}} \circ v_{\lambda}\right)^{*}\left(D \times_{X} X_{\lambda}\right) \geq\left(g_{\lambda} \circ i_{V_{\lambda}} \circ v_{\lambda}\right)^{*}\left(M^{\infty}\right),
$$

similar to (5.4.4). Moreover, letting $\tilde{V}_{\lambda}=h_{\lambda}\left(V_{\lambda}\right)$ with $h_{\lambda}=\left(f_{\lambda}, g_{\lambda}\right): Z_{\lambda} \rightarrow X_{\lambda} \times \bar{M}\left(\tilde{V}_{\lambda}\right.$ is finite over $X_{\lambda}$ by the finiteness of $\left.V_{\lambda} \rightarrow X_{\lambda}\right)$, there exists a dense open immersion $X_{\lambda} \hookrightarrow \overline{X_{\lambda}}$ with $\overline{X_{\lambda}}$ proper such that the closure $\overline{\tilde{V}_{\lambda}}$ of $\tilde{V}_{\lambda}$ in $\overline{X_{\lambda}} \times \bar{M}$ is proper over $\overline{X_{\lambda}}$.

Let $L^{\tau}(Z)$ be the free abelian group on the set $E^{\tau}(Z)$.

Lemma 5.4.2. Let $V_{\lambda}$ be as in (*) $)_{\lambda}$ and $X_{\mu} \rightarrow X_{\lambda}(\lambda, \mu \in \Lambda)$ be a map in the system of étale neighborhoods of $x \in X$. Let

$$
X_{\mu} \stackrel{f_{\mu}}{\longleftarrow} Z_{\mu} \stackrel{g_{\mu}}{\longrightarrow} \bar{M} \quad\left(\text { resp. } V_{\mu} \hookrightarrow Z_{\mu}\right)
$$

be the base change of (5.4.5) (resp. $\left.V_{\lambda} \hookrightarrow Z_{\lambda}\right)$. If $V_{\lambda} \subset Z_{\lambda}$ satisfies $(*)_{\lambda}$, then any component of $V_{\mu}$ satisfies $(*)_{\mu}$.

Proof. The finiteness over $X_{\mu}$ and the admissibility condition of $(\boldsymbol{*})_{\mu}$ are clearly satisfied. To check the last condition of $(\boldsymbol{*})_{\mu}$, let $X_{\mu} \hookrightarrow \overline{X_{\mu}}$ be the normalization in $X_{\mu}$ of $\overline{X_{\lambda}}$ from $(\boldsymbol{*})_{\lambda}$ and let $\tilde{V}_{\mu}=h_{\mu}\left(V_{\mu}\right)$ with $h_{\mu}=\left(f_{\mu}, g_{\mu}\right): Z_{\mu} \rightarrow X_{\mu} \times \bar{M}\left(\tilde{V}_{\mu}\right.$ is finite over $X_{\mu}$ by the finiteness of $\left.V_{\mu} \rightarrow X_{\mu}\right)$. Then $\tilde{V}_{\mu} \subset \tilde{V}_{\lambda} \times_{X_{\lambda}} X_{\mu}$ so that the closure $\overline{V_{\mu}}$ of $\tilde{V}_{\mu}$ in $\overline{X_{\mu}} \times \bar{M}$ is contained in $\overline{\tilde{V}_{\lambda}} \times \overline{X_{\lambda}} \overline{X_{\mu}}$, which is proper over $\overline{X_{\mu}}$ by the assumption. Hence $\overline{V_{\mu}}$ is also proper over $\overline{X_{\mu}}$, which implies the desired condition.

Lemma 5.4.3. For a commutative diagram (5.4.3), there is a natural induced map

$$
\varphi_{*}: E^{\tau}(Z) \rightarrow E^{\tau}\left(Z^{\prime}\right)
$$

which makes $E^{\tau}$ a covariant functor on $\mathcal{D}$.

Proof. Take $V \in E(Z)$ and let $V^{\prime}=\varphi(V)$. By the finiteness of $V \rightarrow \bar{S}, V^{\prime}$ is finite over $\bar{S}$ and closed in $Z^{\prime}$. The admissibility condition (5.4.4) for $V$ implies that for $V^{\prime}$ by [KMSY21, Lemma 1.2.1]. Hence $V^{\prime} \in E\left(Z^{\prime}\right)$. Suppose $V \in E^{\tau}(Z)$. To show $V^{\prime} \in E^{\tau}\left(Z^{\prime}\right)$, take $\lambda \in \Lambda$ and $V_{\lambda}$ as in (\$) ${ }_{\lambda}$. Thanks to Lemma 5.4.2, we may assume that the diagram (5.4.3) is the base change via $\bar{S} \rightarrow X_{\lambda}$ of

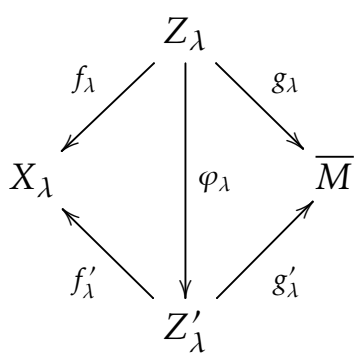

and $V^{\prime}=V_{\lambda}^{\prime} \times_{X_{\lambda}} \bar{S}$ with $V_{\lambda}^{\prime}=\varphi_{\lambda}\left(V_{\lambda}\right)$. Since $V_{\lambda}$ is finite and surjective over a component of $X_{\lambda}$, so is $V_{\lambda}^{\prime}$, which implies that it is an irreducible component of $Z_{\lambda}^{\prime}$. The admissibility condition (5.4.6) for $V_{\lambda}$ implies that for $V_{\lambda}^{\prime}$ by [KMSY21, Lemma 1.2.1]. Letting $h_{\lambda}^{\prime}=\left(f_{\lambda}^{\prime}, g_{\lambda}^{\prime}\right): Z_{\lambda}^{\prime} \rightarrow X_{\lambda} \times \bar{M}$, we have $h_{\lambda}=h_{\lambda}^{\prime} \varphi_{\lambda}$ so that $h_{\lambda}^{\prime}\left(V_{\lambda}^{\prime}\right)=h_{\lambda}\left(V_{\lambda}\right)$. Hence $V_{\lambda}^{\prime}$ satisfies the last condition of $(*)_{\lambda}$ since $V_{\lambda}$ does. This implies $V^{\prime} \in E^{\tau}\left(Z^{\prime}\right)$.

Proof of Theorem 5.4.1(1). It suffices to show the exactness of

$$
\cdots \rightarrow \mathbb{Z}_{\mathrm{tr}}^{\mathrm{fin}}\left(U \times_{M} U\right)^{\tau}(S) \rightarrow \mathbb{Z}_{\mathrm{tr}}^{\mathrm{fin}}(U)^{\tau}(S) \rightarrow \mathbb{Z}_{\mathrm{tr}}^{\mathrm{fin}}(M)^{\tau}(S) \rightarrow 0
$$

where $S=\left(\bar{S}, D \times_{X} \bar{S}\right)$ with $(X, D)$ and $\bar{S}$ as in (5.4.1). We first note that for a closed subscheme $Z \subset$ $\bar{S} \times \bar{U} \times \bar{M} \cdots \times \bar{M} \bar{U}$ finite and surjective over an irreducible component of $\bar{S}$, the image of $Z$ in $\bar{S} \times \bar{M}$ is finite 
over $\bar{S}$. From this fact we see that (5.4.8) is obtained as the inductive limit of

$$
\cdots \rightarrow L^{\tau}(Z \times \bar{M}(\bar{U} \times \bar{M} \bar{U})) \rightarrow L^{\tau}(Z \times \bar{M} \bar{U}) \rightarrow L^{\tau}(Z) \rightarrow 0
$$

where $Z$ ranges over all closed subschemes of $\bar{S} \times \bar{M}$ that is finite surjective over an irreducible component of $\bar{S}$. It suffices to show the exactness of (5.4.9).

Since $Z$ is finite over a henselian local scheme $\bar{S}, Z$ is a disjoint union of henselian local schemes. Thus the Nisnevich cover $Z \times \bar{M} \bar{U} \rightarrow Z$ admits a section $s_{0}: Z \rightarrow Z \times \bar{M} \bar{U}$. Define for $k \geq 0$

$$
s_{k}:=s_{0} \times \bar{M}_{\bar{U}} \operatorname{Id}_{\bar{U}^{k}}: Z \times \bar{M} \bar{U}^{k} \rightarrow Z \times \bar{M} \bar{U} \times \bar{M} \bar{U}^{k}=Z \times \bar{M}_{\bar{M}} \bar{U}^{k+1},
$$

where $\bar{U}^{k}$ is the $k$-fold fiber product of $\bar{U}$ over $\bar{M}$. Then the maps

$$
\left(s_{k}\right)_{*}: L^{\tau}\left(Z \times \bar{M}^{k}\right) \rightarrow L^{\tau}\left(Z \times \bar{M} \bar{U}^{k+1}\right)
$$

give us a homotopy from the identity to zero.

\subsection{Proof of Theorem 5.1.1}

\section{Corollary 5.5.1.}

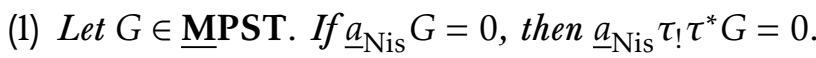

(2) The base change morphism $a_{\mathrm{Nis}} \tau^{*} \Rightarrow \tau^{\mathrm{Nis}} \underline{a}_{\mathrm{Nis}}$ is an isomorphism.

(3) The functor $\tau^{\mathrm{Nis}}$ is exact.

Proof. (1) Since $\underline{a}_{\mathrm{Nis}}, \tau_{\text {! }}$ and $\tau^{*}$ all commute with representable colimits as left adjoints, we are reduced by Lemma 5.2.1 to $G$ of the form $\mathbb{Z}_{\text {tr }}(M / U)$, which is equivalent to

$$
\underline{a}_{\mathrm{Nis}}\left(\operatorname{Coker}\left(\mathbb{Z}_{\mathrm{tr}}(U)^{\tau} \rightarrow \mathbb{Z}_{\mathrm{tr}}(M)^{\tau}\right)\right)=0,
$$

where the cokernel is taken in MPST. This follows from Theorem 5.4.1(2).

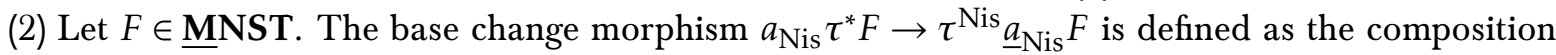

$$
a_{\mathrm{Nis}} \tau^{*} F \stackrel{a_{\mathrm{Nis}} \tau^{*}\left(\eta_{F}\right)}{\longrightarrow} a_{\mathrm{Nis}} \tau^{*} \underline{\underline{N}}_{\mathrm{Nis}} \underline{a}_{\mathrm{Nis}} F \simeq a_{\mathrm{Nis}} i_{\mathrm{Nis}} \tau^{\mathrm{Nis}} \underline{a}_{\mathrm{Nis}} F \stackrel{\varepsilon^{\mathrm{Nis}} \underline{\mathrm{N}}_{\mathrm{Nis}} F}{\longrightarrow} \tau^{\mathrm{Nis}} \underline{a}_{\mathrm{Nis}} F
$$

where $\eta($ resp. $\varepsilon)$ is the unit (resp. counit) of the adjunction $\left(\underline{a}_{\mathrm{Nis}}, \underline{i}_{\mathrm{Nis}}\right)$ (resp. $\left.\left(a_{\mathrm{Nis}}, i_{\mathrm{Nis}}\right)\right)$. Since the second map is an isomorphism by the full faithfulness of $i_{\mathrm{Nis}}$, it remains to show that the first one is an isomorphism. By the full faithfulness of $\tau_{\text {Nis }}$ (Lemma 4.2.5), it suffices to show it after applying this functor. But $\underline{a}_{\mathrm{Nis}} \tau_{!} \simeq \tau_{\mathrm{Nis}} a_{\mathrm{Nis}}$ by Theorem 4.2.4, so we are left to show that the map

$$
\underline{a}_{\mathrm{Nis}} \tau_{!} \tau^{*} F \stackrel{\underline{a}_{\mathrm{Nis}} \tau_{!} \tau^{*}\left(\eta_{F}\right)}{\longrightarrow} \underline{a}_{\mathrm{Nis}} \tau_{!} \tau^{*} \underline{i}_{\mathrm{Nis}} \underline{\mathrm{a}}_{\mathrm{Nis}} F
$$

is an isomorphism. This follows from (1), since $\underline{a}_{\mathrm{Nis}} \tau_{!} \tau^{*}$ is exact, and Ker $\eta_{F}$ and Coker $\eta_{F}$ are killed by $\underline{a}_{\mathrm{Nis}}$.

Finally, (3) follows from (2), Lemma 1.2.6 (4) and the exactness of $\tau^{*}$.

Corollary 5.5.2. The functor $\tau^{\mathrm{Nis}}$ has a right adjoint.

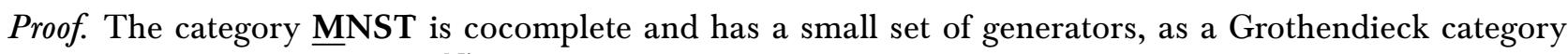
(Theorem 2.2.7.) Moreover, $\tau^{\mathrm{Nis}}$ respects all representable colimits as an exact, strongly additive functor (Lemma 4.2.5 and Corollary 5.5.1 (3)). Thus the dual hypotheses of the "special adjoint functor theorem" [Mac98, Chapter V, Section 8, Theorem 2] are verified.

This corollary is striking, since $\tau_{s}$ is not cocontinuous [KM20, Remark 5.2.1]. 


\section{Relation with NST}

\subsection{MNS, MNS and NS}

We consider the functors

$$
\underline{\omega}_{S}: \underline{\mathbf{M S m}} \rightarrow \mathbf{S m}, \quad \omega_{s}: \mathbf{M S m} \rightarrow \mathbf{S m},
$$

defined by $\underline{\omega}_{s}(M)=M^{\mathrm{o}}$ and $\omega_{s}(M)=M^{\mathrm{o}}$, and the left adjoint to $\underline{\omega}_{s}$, defined by $\lambda_{s}(X)=(X, \emptyset)$. We have $\omega_{s}=\underline{\omega}_{s} \tau_{s}$, and:

Proposition 6.1.1 ([KM20, Theorem 1]). The functors $\omega_{s}: \mathbf{M S m}_{\mathrm{Nis}} \rightarrow \mathbf{S m}_{\mathrm{Nis}}, \underline{\omega}_{s}: \underline{\mathbf{M S m}_{\mathrm{Nis}}} \rightarrow \mathbf{S m}_{\mathrm{Nis}}$ and $\lambda_{s}: \mathrm{Sm} \rightarrow \underline{\mathrm{MSm}}$ are continuous and cocontinuous.

Let PS (resp. NS) be the category of abelian presheaves (resp. Nisnevich sheaves) on Sm. The inclusion $i_{s, \mathrm{Nis}}^{V}:$ NS $\hookrightarrow$ PS has a left adjoint $a_{s, \mathrm{Nis}}^{V}$. Let $\underline{\omega}_{s}^{*}:$ PS $\rightarrow$ MPS and $\omega_{s}^{*}:$ PS $\rightarrow$ MPS be the functors induced by $\underline{\omega}_{S}$ and $\omega_{s}$. They have left adjoints $\underline{\omega}_{s, !}$ and $\omega_{s, !}$.

\section{Proposition 6.1.2.}

a) We have $\underline{\omega}_{s, !}(\underline{\mathbf{M N S}}) \subset \mathbf{N S}$ and $\omega_{s, !}(\mathbf{M N S}) \subset \mathbf{N S}$.

b) For $F \in \mathbf{P S}, \omega_{S}^{*} F \in \mathbf{M N S} \Longleftrightarrow \underline{\omega}_{S}^{*} F \in \underline{\mathbf{M N S}} \Longleftrightarrow F \in \mathbf{N S}$.

c) Let $\omega_{s}^{\mathrm{Nis}}: \mathrm{NS} \rightarrow \mathrm{MNS}$ and $\omega_{s, \mathrm{Nis}}: \mathrm{MNS} \rightarrow \mathrm{NS}$ be the functors such that

$$
i_{s, \mathrm{Nis}} \omega_{s}^{\mathrm{Nis}}=\omega_{s}^{*} i_{s, \mathrm{Nis}}^{V}, \quad i_{s, \mathrm{Nis}}^{V} \omega_{s, \mathrm{Nis}}=\omega_{s, !} i_{s, \mathrm{Nis}},
$$

which exist by b). Then $\omega_{s, \mathrm{Nis}}$ is left adjoint to $\omega_{s}^{\mathrm{Nis}}$; both functors are exact and $\omega_{s}^{\mathrm{Nis}}$ is strongly additive. We have $\omega_{s, \mathrm{Nis}}=a_{s, \mathrm{Nis}}^{V} \omega_{s, !} i_{s, \mathrm{Nis}}$ and

$$
\omega_{s, \mathrm{Nis}} a_{s, \mathrm{Nis}}=a_{s, \mathrm{Nis}}^{V} \omega_{s, !}, \quad \omega_{s}^{\mathrm{Nis}} a_{s, \mathrm{Nis}}^{V}=a_{s, \mathrm{Nis}} \omega_{s}^{*} .
$$

d) Let $\underline{\omega}_{s}^{\mathrm{Nis}}: \mathrm{NS} \rightarrow \underline{\mathrm{MNS}}$ and $\underline{\omega}_{s, \mathrm{Nis}}: \underline{\mathrm{MNS}} \rightarrow \mathrm{NS}$ be the functors defined in the same way as $\omega_{s}^{\mathrm{Nis}}$ and $\omega_{s, \mathrm{Nis}}$ in c). Then the similar statements to c) hold for $\underline{\omega}_{s}^{\mathrm{Nis}}$ and $\underline{\omega}_{s, \mathrm{Nis}}$.

Proof. a) Since $\lambda_{s}$ is left adjoint to $\underline{\omega}_{s}$, we have $\underline{\omega}_{s, !}=\lambda_{s}^{*}$, hence the continuity of $\lambda_{s}$ proves the first assertion. The second one follows from Theorem 4.1.2, as $\omega_{s, !}=\underline{\omega}_{s, !} \tau_{s, !}$.

b) If $\underline{\omega}_{s}^{*} F \in \underline{\mathbf{M N S}}$, then $\omega_{s}^{*} F=\tau_{s}^{*} \underline{\omega}_{s}^{*} F \in \mathbf{M N S}$ by Theorem 4.1.2. If $\omega_{s}^{*} F \in \mathbf{M N S}$, then $\omega_{s, !} \omega_{s}^{*} F \stackrel{\sim}{\rightarrow} F \in \mathbf{N S}$ by a) since $\omega_{S}^{*}$ is fully faithful. If $F \in \mathbf{N S}$, then we have $\underline{\omega}_{S}^{*} F \in \underline{\mathbf{M N S}}$ since $\underline{\omega}_{S}$ is continuous.

c) The second formula of (6.1.3) follows from the cocontinuity of $\omega_{s}$ (Proposition 6.1.1) and [SGA4, exposé III, Proposition 2.3 (2)]. We prove the rest of the assertions by using Lemma 1.2.6 as follows. In the situation of Lemma 1.2.6, set $\mathcal{C}=\mathbf{P S}, \mathcal{C}^{\prime}=\mathrm{NS}, \mathcal{D}=\operatorname{MPS}, \mathcal{D}^{\prime}=\operatorname{MNS},\left(i_{\mathcal{C}}, i_{\mathcal{D}}\right)=\left(i_{s, \mathrm{Nis}}^{V}, i_{s, \mathrm{Nis}}\right)$ and $\left(c, c^{\prime}, d\right)=\left(\omega_{s}^{*}, \omega_{s}^{\mathrm{Nis}}, \omega_{s, !}\right)$.

The assumption of Lemma 1.2.6 (2) is satisfied since $i_{s, \mathrm{Nis}}$ is strongly additive by Theorem 2.2.7, $i_{s, \mathrm{Nis}}^{V}$ is strongly additive by the quasi-compactness of the Nisnevich topology, and $\omega_{s}^{*}$ is strongly additive as a left adjoint. Hence $\omega_{s}^{\mathrm{Nis}}$ is also strongly additive.

The assumption of Lemma 1.2.6 (3) is satisfied since $i_{s, \mathrm{Nis}}^{V}, i_{s, \mathrm{Nis}}$ have exact left adjoints $a_{s, \mathrm{Nis}}^{V}, a_{s, \mathrm{Nis}}$, and since $\omega_{s, !}$ is exact by [KMSY21, Proposition 2.2.1]. Hence the left adjoint $\omega_{s, \mathrm{Nis}}$ of $\omega_{s}^{\mathrm{Nis}}$ is exact, and we have $\omega_{s, \mathrm{Nis}}=a_{s, \mathrm{Nis}}^{V} \omega_{s, !} i_{s, \mathrm{Nis}}$ and $a_{s, \mathrm{Nis}}^{V} \omega_{s, !}=\omega_{s, \mathrm{Nis}} a_{s, \mathrm{Nis}}$.

The assumption of Lemma 1.2.6 (4) is satisfied. Indeed, the formula $a_{\mathcal{D}} c=c^{\prime} a_{\mathcal{C}}$ coincides with the second formula of (6.1.3) (which we have proven above), and $\omega_{s}^{*}$ is exact as a left and right adjoint. Hence $\omega_{s}^{\text {Nis }}$ is exact.

d) is shown by the same argument as c), by the cocontinuity of $\underline{\omega}_{S}$ (Proposition 6.1.1) and by Lemma 1.2.6 applied to $\mathcal{C}=\mathbf{P S}, \mathcal{C}^{\prime}=\mathbf{N S}, \mathcal{D}=\underline{\operatorname{MPS}}, \mathcal{D}^{\prime}=\underline{\operatorname{MNS}},\left(i_{\mathcal{C}}, i_{\mathcal{D}}\right)=\left(i_{s, \mathrm{Nis}}^{V}, \underline{i}_{s, \mathrm{Nis}}\right)$ and $\left(c, c^{\prime}, d\right)=\left(\underline{\omega}_{s}^{*}, \underline{\omega}_{s}^{\mathrm{Nis}}, \underline{\omega}_{s, !}\right)$. 
Indeed, $\underline{i}_{s, \mathrm{Nis}}$ is strongly additive (Lemma 4.1.1), $\underline{\omega}_{S}^{*}$ is exact as a left and right adjoint, $\underline{i}_{s, \text { Nis }}$ has an exact left adjoint $\underline{a}_{s, \mathrm{Nis}}$, and $\underline{\omega}_{s, !}$ is exact. This finishes the proof.

\subsection{MNST, $\underline{M} S S T$ and NST}

Let PST be the abelian category of presheaves on Cor. The graph functor $c^{V}: \mathrm{Sm} \rightarrow$ Cor induces an exact faithful functor $c^{V *}:$ PST $\rightarrow$ PS. Let NST be the full subcategory of PST consisting of $F \in$ PST such that $c^{V *} F \in \mathbf{N S}$. The functor $c^{V *}$ restricts to $c^{V \text {,Nis }}:$ NST $\rightarrow$ NS. The inclusion $i_{\text {Nis }}^{V}:$ NST $\hookrightarrow$ PST has a left adjoint $a_{\mathrm{Nis}}^{V}$ by [Voe00, Theorem 3.1.4]. By construction, it satisfies

$$
c^{V, \mathrm{Nis}} a_{\mathrm{Nis}}^{V}=a_{s, \mathrm{Nis}}^{V} c^{V *} .
$$

We consider the functors

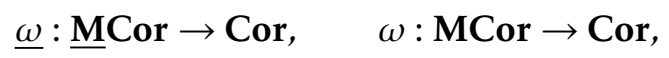

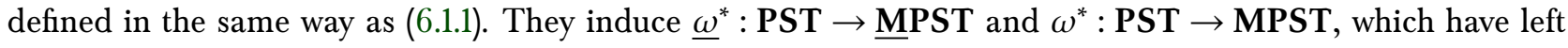
adjoints $\underline{\omega}_{!}$and $\omega_{!}$. One has the obvious identifications

$$
\underline{c}^{*} \underline{\omega}^{*}=\underline{\omega}_{S}^{*} c^{V^{*}}, \quad c^{*} \omega^{*}=\omega_{s}^{*} c^{V *} .
$$

One also sees from [KMSY21, (2.2.1)] (and its analogues for $\omega_{s}, \underline{\omega}_{s}$ ) that

$$
c^{V *} \underline{\omega}_{!}=\underline{\omega}_{s, !} \underline{c}^{*}, \quad c^{V *} \omega_{!}=\omega_{s, !} c^{*} .
$$

\section{Proposition 6.2.1.}

a) We have $\underline{\omega}_{!}(\underline{\text { MNST }}) \subset$ NST and $\omega_{!}(\mathbf{M N S T}) \subset \mathbf{N S T}$.

b) For $F \in \mathbf{P S T}, \omega^{*} F \in \mathbf{M N S T} \Longleftrightarrow \underline{\omega}^{*} F \in \underline{\mathbf{M N S T}} \Longleftrightarrow F \in \mathbf{N S T}$.

c) Let $\omega^{\mathrm{Nis}}: \mathrm{NST} \rightarrow \mathrm{MNST}$ and $\omega_{\mathrm{Nis}}:$ MNST $\rightarrow$ NST be the functors such that

$$
i_{\mathrm{Nis}} \omega^{\mathrm{Nis}}=\omega^{*} i_{\mathrm{Nis}}^{V}, \quad i_{\mathrm{Nis}}^{V} \omega_{\mathrm{Nis}}=\omega_{!} i_{\mathrm{Nis}},
$$

where the second equality shows that $\omega_{\mathrm{Nis}}=a_{\mathrm{Nis}}^{V} \omega_{!} i_{\mathrm{Nis}}$ by $a_{\mathrm{Nis}}^{V} i_{\mathrm{Nis}}^{V}=\mathrm{Id}$. Then $\omega_{\mathrm{Nis}}$ is left adjoint to $\omega^{\mathrm{Nis}}$, and we have

$$
\begin{aligned}
& \omega_{\mathrm{Nis}} a_{\mathrm{Nis}}=a_{\mathrm{Nis}}^{V} \omega_{!}, \quad \omega^{\mathrm{Nis}} a_{\mathrm{Nis}}^{V}=a_{\mathrm{Nis}} \omega^{*}, \\
& c^{\mathrm{Nis}} \omega^{\mathrm{Nis}}=\omega_{s}^{\mathrm{Nis}} c^{V, \mathrm{Nis}} .
\end{aligned}
$$

Moreover, the functors $\omega_{\mathrm{Nis}}$ and $\omega^{\mathrm{Nis}}$ are both exact, $\omega^{\mathrm{Nis}}$ is fully faithful, strongly additive and preserves injectives.

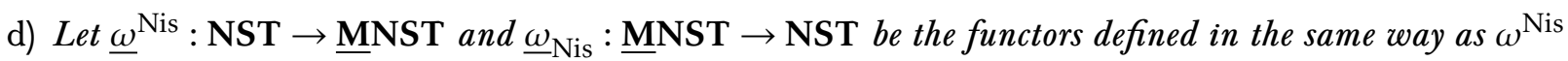
and $\omega_{\mathrm{Nis}}$ in c). Then the similar statements as $c$ ) holds for $\underline{\omega}^{\mathrm{Nis}}$ and $\underline{\omega}_{\mathrm{Nis}}$.

Proof. a) First we prove $\underline{\omega}_{!}(\underline{\text { MNST }}) \subset$ NST. Since $\underline{\underline{c}} \underline{ }(\underline{\text { MNST}}) \subset \underline{\text { MNS }}$ (see Lemma-Definition 2.2.5), we have

$$
c^{V *} \underline{\omega}_{!}(\underline{\mathbf{M N S T}})=\underline{\omega}_{s, !} \underline{c}^{*}(\underline{\mathbf{M N S T}}) \subset \underline{\omega}_{s, !}(\underline{\mathbf{M N S}}) \subset \mathbf{N S},
$$

where the first equality follows from the first equality of (6.2.4), and the last inclusion follows from Proposition 6.1 .2 a). Then by definition of NST we have $\underline{\omega}_{!}(\underline{\text { MNST }}) \subset$ NST, as desired. The inclusion $\omega_{!}($MNST $) \subset$ NST follows from this and Lemma-Definition 4.2.1.

b) follows from Proposition 6.1.2 b), (6.2.4) and the definitions of NST, $\underline{\text { MNST and MNST. }}$

c) The full faithfulness of $\omega^{\text {Nis }}$ follows from that of $\omega^{*}$ [KMSY21, Proposition 2.2.1], and (6.2.7) follows from (6.2.3). The strategy of the rest of the proof is similar to that of $\mathrm{c}$ ) in Proposition 6.1.2. In the situation of Lemma 1.2.6, set $\mathcal{C}=$ PST, $\mathcal{C}^{\prime}=$ NST, $\mathcal{D}=\operatorname{MPST}, \mathcal{D}^{\prime}=\operatorname{MNST},\left(i_{\mathcal{C}}, i_{\mathcal{D}}\right)=\left(i_{\mathrm{Nis}}^{V}, i_{\mathrm{Nis}}\right)$ and $\left(c, c^{\prime}, d\right)=\left(\omega^{*}, \omega^{\mathrm{Nis}}, \omega_{!}\right)$. 
The assumptions of Lemma 1.2.6 (2) and (3) are satisfied, since $i_{\mathrm{Nis}}$ is strongly additive by Theorem 4.2.4 $(1), i_{\text {Nis }}^{V}$ is strongly additive by the quasi-compactness of Nisnevich cohomology, $\omega^{*}$ is strongly additive as a left and right adjoint, $i_{\mathrm{Nis}}^{V}, i_{\mathrm{Nis}}$ have exact left adjoints $a_{\mathrm{Nis}}^{V}, a_{\mathrm{Nis}}$ by [MVW06] and 4.2.4 (2), and $\omega_{\text {! }}$ is exact by [KMSY21, Proposition 2.2.1]. Therefore, the assertions follow, except for the second identity of (6.2.6) and the exactness of $\omega^{\text {Nis }}$. (Note that the exactness of $\omega_{\text {Nis }}$ implies that $\omega^{\text {Nis }}$ preserves injectives.)

We can prove the second identity of (6.2.6) as follows. Its first identity yields a base change morphism

$$
a_{\mathrm{Nis}} \omega^{*} \Rightarrow \omega^{\mathrm{Nis}} a_{\mathrm{Nis}}^{V}
$$

Let $F \in \mathbf{P S T}$. We want to show that the morphism $a_{\mathrm{Nis}} \omega^{*} F \rightarrow \omega^{\mathrm{Nis}} a_{\mathrm{Nis}}^{V} F$ is an isomorphism. Since $c^{\mathrm{Nis}}$ is conservative as MSm and MCor have the same objects, it suffices to show that the induced morphism $c^{\mathrm{Nis}} a_{\mathrm{Nis}} \omega^{*} F \rightarrow c^{\mathrm{Nis}} \omega^{\mathrm{Nis}} a_{\mathrm{Nis}}^{V} F$ is an isomorphism. Since

$$
\begin{gathered}
c^{\mathrm{Nis}} a_{\mathrm{Nis}} \omega^{*} \stackrel{(4.2 .3)}{=} a_{s, \mathrm{Nis}} c^{*} \omega^{*} \stackrel{(6.2 .3)}{=} a_{s, \mathrm{Nis}} \omega_{s}^{*} c^{V *}, \\
c^{\mathrm{Nis}} \omega^{\mathrm{Nis}} a_{\mathrm{Nis}}^{V} \stackrel{(6.2 .7)}{=} \omega_{s}^{\mathrm{Nis}} c^{V, \mathrm{Nis}} a_{\mathrm{Nis}}^{V} \stackrel{(6.2 .1)}{=} \omega_{s}^{\mathrm{Nis}} a_{s, \mathrm{Nis}}^{V} c^{V *},
\end{gathered}
$$

the above morphism is rewritten as

$$
a_{s, \mathrm{Nis}} \omega_{s}^{*} c^{V *} F \rightarrow \omega_{s}^{\mathrm{Nis}} a_{s, \mathrm{Nis}}^{V} c^{V *} F
$$

which is an isomorphism by Proposition $6.1 .2 \mathrm{c}$ ).

Now, the formula we have proven and the exactness of $\omega^{*}$ as a left and right adjoint show that the assumption of Lemma 1.2.6 (4) is satisfied. Hence $\omega^{\text {Nis }}$ is exact, as desired. This finishes the proof of c).

d) The proof is completely parallel to that of c). To see this, it suffices to observe the following: $\underline{i}_{\mathrm{Nis}}$ is strongly additive and has an exact left adjoint by [KMSY21, Lemma 4.5.3, Theorem 4.5.5], $\underline{\omega}^{*}$ is strongly additive as a left and right adjoint, $\underline{\omega}_{!}$is exact by [KMSY21, Proposition 2.3.1], $\underline{c}^{\text {Nis }}$ is conservative since

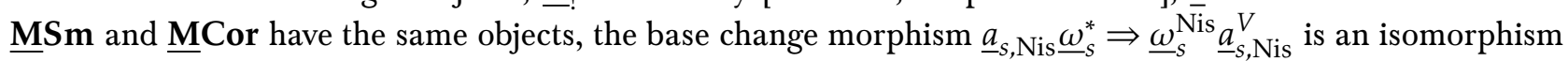
by Proposition $6.1 .2 \mathrm{~d}$ ), and we have the following identifications:

$$
\begin{gathered}
\underline{c}^{\mathrm{Nis}} \underline{\underline{a}}_{\mathrm{Nis}} \underline{\omega}^{*} \stackrel{(2.2 .3)}{=} \underline{a}_{s, \mathrm{Nis}} \underline{c}^{*} \underline{\omega}^{*} \stackrel{(6.2 .3)}{=} \underline{a}_{s, \mathrm{Nis}} \underline{\omega}_{s}^{*} c^{V *}, \\
\underline{c}^{\mathrm{Nis}} \underline{\omega}^{\mathrm{Nis}} a_{\mathrm{Nis}}^{V} \stackrel{(6.2 .7)}{=} \underline{\omega}_{s}^{\mathrm{Nis}} c^{V, \mathrm{Nis}} a_{\mathrm{Nis}}^{V} \stackrel{(6.2 .1)}{=} \underline{\omega}_{s}^{\mathrm{Nis}} a_{s, \mathrm{Nis}}^{V} c^{V *} .
\end{gathered}
$$

This finishes the proof.

\subsection{Relation between cohomologies}

We now prove Theorem 3 (2) from the introduction.

Lemma 6.3.1. Let $I \in$ MNST be an injective object. Then $\omega_{\mathrm{Nis}} I \in \mathrm{NST}$ is flasque.

Proof. Let $U \rightarrow X$ in Sm be an open dense immersion. We need to show the surjectivity of

$$
\omega_{\mathrm{Nis}} I(X)=\underset{M \in \mathbf{M S m}(X)}{\lim } I(M) \rightarrow \omega_{\mathrm{Nis}} I(U)=\underset{N \in \underset{\mathbf{M S m}}{\longrightarrow}(U)}{\lim } I(U) .
$$

We fix $M \in \operatorname{MSm}(X)$ and will show that the composition $I(M) \rightarrow \omega_{\mathrm{Nis}} I(X) \rightarrow \omega_{\mathrm{Nis}} I(U)$ is already surjective. This follows from the functoriality of $\omega^{!}$, but for clarity we give an explicit argument. Take any $N \in \mathbf{M S m}(U)$. Let $\bar{N}^{\prime}$ be the closure of the image of $U \rightarrow \bar{N} \times \bar{M}$, and let $\bar{L}$ be the blow-up of $\bar{N}^{\prime}$ along $\left(\bar{N}^{\prime} \backslash U\right)_{\text {red. }}$. Set $L:=\left(\bar{L}, L^{\infty}\right) \in$ MCor where $L^{\infty}$ is the pull-back of $N^{\infty}$ along the composition $\bar{L} \rightarrow \bar{N}^{\prime} \rightarrow \bar{N}$. We have a commutative diagram in $\underline{\text { MCor }}$

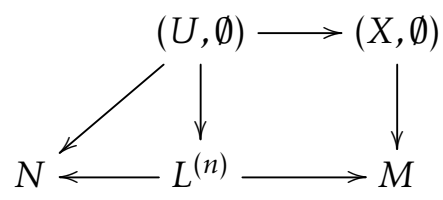


for sufficiently large $n$ (see [KMSY21, Definition 1.4.1]). Since $U \rightarrow X$ is an open dense immersion, $\operatorname{Cor}(V, U) \rightarrow \operatorname{Cor}(V, X)$ is injective for any $V \in \mathbf{S m}$, which in turn implies the injectivity of the morphism $\mathbb{Z}_{\mathrm{tr}}\left(L^{(n)}\right) \rightarrow \mathbb{Z}_{\mathrm{tr}}(M)$ in MNST. Since $I$ is an injective object, we conclude that $I(M) \rightarrow I\left(L^{(n)}\right)$ is surjective. This proves the lemma, as the canonical map $I(N) \rightarrow \omega_{\mathrm{Nis}} I(U)$ factors through $I\left(L^{(n)}\right)$.

\section{Theorem 6.3.2.}

(1) For any $M \in$ MCor, $G \in \mathrm{NST}$ and $p \geq 0$, we have a canonical isomorphism

$$
\operatorname{Ext}_{\text {MNST }}^{p}\left(\mathbb{Z}_{\mathrm{tr}}(M), \omega^{\mathrm{Nis}} G\right) \cong H_{\mathrm{Nis}}^{p}\left(M^{\mathrm{o}}, G\right) .
$$

(2) For any $X \in \mathbf{S m}, F \in \mathbf{M N S T}$ and $p \geq 0$, we have a canonical isomorphism

$$
H_{\mathrm{Nis}}^{p}\left(X, \omega_{\mathrm{Nis}} F\right) \cong \underset{M \in \mathbb{\operatorname { M S m }}(X)}{\lim _{\mathrm{Nis}}} H_{M}^{p}\left(\bar{M}, F_{M}\right) .
$$

Proof. It follows from Proposition 6.2.1 and Theorem [KMSY21, Theorem A.9.1] that

$$
\left(R^{p} i_{\text {Nis }}\right) \omega^{\mathrm{Nis}}=R^{p}\left(i_{\mathrm{Nis}} \omega^{\mathrm{Nis}}\right)=R^{p}\left(\omega^{*} i_{\mathrm{Nis}}^{V}\right)=\omega^{*} R^{p} i_{\mathrm{Nis}}^{V} .
$$

By Proposition A.1.1 and the projectivity of $\mathbb{Z}_{\mathrm{tr}}(M)$ and $\mathbb{Z}_{\mathrm{tr}}^{V}\left(M^{\mathrm{o}}\right)$, we get

$$
\begin{aligned}
& \operatorname{Ext}_{\text {MNST }}^{p}\left(\mathbb{Z}_{\mathrm{tr}}(M), \omega^{\mathrm{Nis}} G\right) \cong \operatorname{MPST}\left(\mathbb{Z}_{\mathrm{tr}}(M),\left(R^{p} i_{\mathrm{Nis}}\right) \omega^{\mathrm{Nis}} G\right) \\
& \cong \operatorname{MPST}\left(\mathbb{Z}_{\mathrm{tr}}(M), \omega^{*} R^{p} i_{\mathrm{Nis}}^{V} G\right) \cong \operatorname{PST}\left(\omega_{!} \mathbb{Z}_{\mathrm{tr}}(M), R^{p} i_{\mathrm{Nis}}^{V} G\right) \\
& \cong \operatorname{PST}\left(\mathbb{Z}_{\mathrm{tr}}^{V}\left(M^{\mathrm{o}}\right), R^{p} i_{\mathrm{Nis}}^{V} G\right) \cong H_{\mathrm{Nis}}^{p}\left(M^{\mathrm{o}}, G\right),
\end{aligned}
$$

whence (1).

Given $X \in \mathbf{S m}$, we define functors $\Gamma_{X}^{\rightarrow}:$ MNST $\rightarrow$ Ab and $\Gamma_{X}^{V}:$ NST $\rightarrow$ Ab by

$$
\Gamma_{X}^{\vec{S}}(F)=\underset{M \in \operatorname{MSm}(X)}{\lim _{\longrightarrow}} F(M), \quad \Gamma_{X}^{V}(G)=G(X) .
$$

We have $\Gamma_{X} \rightarrow=\Gamma_{X}^{V} \omega_{\text {Nis }}$. By [KMSY21, Theorem A.9.1] and Lemma 6.3.1, we get $\left(R^{p} \Gamma_{X}^{V}\right) \omega_{\text {Nis }}=R^{p} \Gamma_{X}$ for any $p \geq 0$, since $\omega_{\mathrm{Nis}}$ is exact. Taking an injective resolution $F \rightarrow I^{\bullet}$ in MNST, we proceed

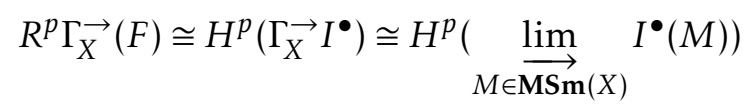

$$
\begin{aligned}
& \cong \underset{M \in \underset{\operatorname{MSm}}{\longrightarrow}(X)}{\lim _{P}} H^{p}\left(I^{\bullet}(M)\right) \quad \text { [KMSY21, Lemma 1.7.4] } \\
& \cong \underset{M \in \operatorname{MSm}(X)}{\lim _{N \in \sum^{\text {fin } \downarrow M}}} \underset{\lim _{\mathrm{Nis}}}{\longrightarrow}\left(\bar{N}, F_{N}\right) \quad \text { (Theorem 5.1.3) } \\
& \cong \lim _{M \in \operatorname{MSm}(X)} H_{\mathrm{Nis}}^{p}\left(\bar{M}, F_{M}\right) \text {. }
\end{aligned}
$$

Here the last isomorphism holds since any $N \in \Sigma^{\text {fin }} \downarrow M$ gives rise to an object $N \in \operatorname{MSm}(X)$. This concludes the proof of (2).

\section{Passage to derived categories}

In this section, we extend the previous results to derived categories. The main result is an extension of Proposition 2.2.9 and Theorem 5.1.3 to unbounded complexes (Proposition 7.4.2 (2) and Theorem 7.5.1 (3)). 


\subsection{Compactness}

Proposition 7.1.1. Let $M \in \underline{\mathbf{M S m}}$. Then the following objects are compact:

(1) $\mathbb{Z}_{\mathrm{tr}}(M)[0]$ in $D(\underline{\mathrm{MNST}})$, and in $D(\mathbf{M N S T})$ if $M$ is proper.

(2) $\mathbb{Z}(M)[0]$ in $D\left(\underline{\mathbf{M N S}}^{\text {fin }}\right)$ and $D(\underline{\mathbf{M N S}})$.

Proof. (1) follows from Proposition 2.2.9 (resp. from Theorem 5.1.3) and the known commutation of Nisnevich cohomology with filtered colimits of sheaves, via hypercohomology spectral sequences which are convergent and Corollary 2.2.10 (resp. 5.1.4). (2) is seen similarly, using [KMSY21, (3.6.1) and Proposition 4.4.2].

\subsection{Strong additivity}

\section{Theorem 7.2.1.}

(1) (cf. [KMSY21, Proposition 4.3.3 (2)]) The functor $R \underline{b}_{s}^{\mathrm{Nis}}: D(\underline{\mathrm{MNS}}) \rightarrow D\left(\underline{M N S}^{\mathrm{fin}}\right)$ is right adjoint to $D\left(\underline{b}_{s, \mathrm{Nis}}\right)$ and is strongly additive. The counit map $D\left(\underline{b}_{s, \mathrm{Nis}}\right) R \underline{b}_{s}^{\mathrm{Nis}} \rightarrow \mathrm{Id}$ is an isomorphism.

(2) The functor $R\left(\underline{b}_{s}^{\mathrm{Nis}} \circ \underline{c}^{\mathrm{Nis}}\right)$ is strongly additive.

Proof. (1) The adjunction statement follows from Proposition A.2.5. The second assertion implies the third by Lemma A.2.7. It remains to prove the strong additivity of $R \underline{b}_{s}^{\text {Nis }}$. For this, we check that the hypothesis of Proposition A.2.8 c) are verified: we take the $\mathbb{Z}(M), M \in \mathbf{M S m}^{\text {fin }}$, as a set of generators. We have $\underline{b}_{s, \mathrm{Nis}} \mathbb{Z}(M)=\mathbb{Z}(M)$. Their compactness follows from Proposition 7.1.1 (2).

(2) Same argument as (1), using Proposition 7.1.1 (1) as well.

\subsection{From $D\left(\underline{M}^{\text {Nin }}\right)$ to $D(\underline{\mathbf{M N S}})$}

We first extend [KMSY21, Notation 4.4.1] from sheaves to complexes of sheaves:

Notation 7.3.1. Let $N \in \underline{\mathbf{M S m}}^{\text {fin }}$ and let $K$ be a complex on $\underline{\mathbf{M N S}}^{\text {fin }}$. We write $K_{N}$ for the complex of sheaves on $(\bar{N})_{\text {Nis }}$ deduced from $K$ via the isomorphism of sites from [KMSY21, Lemma 3.1.3]. If $K$ is a complex on $\underline{\mathrm{MNS}}$, we write $K_{N}$ for $\left(\underline{b}_{s}^{\mathrm{Nis}} K\right)_{N}$.

The following extends [KMSY21, Proposition 4.4.2] to unbounded complexes of sheaves.

Proposition 7.3.2. Let $M \in \underline{\mathbf{M S}} S$. For $K \in D(\underline{\mathbf{M N S}})$, we have natural isomorphisms

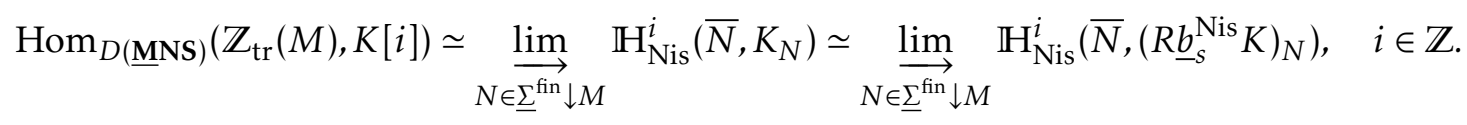

Proof. Define as in loc. cit. functors $\Gamma_{M}^{\downarrow}: \underline{\text { MNS }}{ }^{\text {fin }} \rightarrow \mathbf{A b}$ and $\underline{\Gamma}_{M}: \underline{\text { MNS }} \rightarrow$ Ab by

$$
\Gamma_{M}^{\downarrow}(G)=\underset{N \in \underline{\Sigma}^{\mathrm{fin}} \downarrow M}{\lim } G(N), \quad \underline{\Gamma}_{M}(F)=F(M) .
$$

We have $\Gamma_{M}^{\downarrow}=\underline{\Gamma}_{M} \underline{b}_{s, \mathrm{Nis}}$ and we shall show that the natural transformation (A.2.1)

$$
R \Gamma_{M}^{\downarrow} \Rightarrow R \underline{\Gamma}_{M} \circ D\left(\underline{b}_{s, \mathrm{Nis}}\right)
$$

is invertible. For this, we apply Lemma A.2.7. Its first condition is given by [KMSY21, Lemma 4.4.3], which says that $\underline{b}_{s, \text { Nis }}$ sends injectives to flabbys; by [KMSY21, Theorem A.9.1 and Lemma A.9.3], this already yields isomorphisms

$$
R^{p} \Gamma_{M}^{\downarrow} \stackrel{\sim}{\rightarrow} R^{p} \underline{\Gamma}_{M} \circ \underline{b}_{s, \mathrm{Nis}}, \quad p \geq 0 .
$$

We are now left to show the strong additivity of the three functors. For $D\left(\underline{b}_{s, \mathrm{Nis}}\right)$, this follows from the strong additivity of $\underline{b}_{s, \mathrm{Nis}}$ as a left adjoint, and Proposition A.2.8 a). 
For $R \Gamma_{M}^{\downarrow}$ and $R \underline{\Gamma}_{M}$, we check that the conditions of Proposition A.2.8 b) are verified. For $R \underline{\Gamma}_{M}$, Condition (ii) follows from the vanishing statement in (1) (use the compact projective generator $\mathbb{Z}$ of $\mathbf{A b}$ ), and Condition (i) follows similarly from the known commutation of Nisnevich cohomology with filtered colimits of sheaves. The case of $R \Gamma_{M}^{\downarrow}$ is reduced to this one by (7.3.2).

Thus we get an isomorphism

$$
\operatorname{Hom}_{D(\underline{\mathbf{M N S}})}\left(\mathbb{Z}_{\mathrm{tr}}(M), \underline{b}_{s, \mathrm{Nis}} L[i]\right) \simeq \underset{N \in \underline{\underline{\Sigma}}^{\mathrm{fin}} \downarrow M}{\lim _{\mathrm{Nis}}} \mathbb{H}_{\mathrm{Nis}}^{i}\left(\bar{N}, L_{N}\right), \quad i \in \mathbb{Z}
$$

for any complex $L$ on $\underline{\text { MNS }}{ }^{\text {fin }}$. Setting $L=\underline{b}_{s}^{\text {Nis }} K$, we get the first isomorphism thanks to the isomorphism $\underline{b}_{s, \mathrm{Nis}} \underline{b}_{s}^{\mathrm{Nis}} \stackrel{\sim}{\rightarrow}$ Id of [KMSY21, Proposition 4.3.3 (2)]. Composing (7.3.1) with $R \underline{b}_{s}^{\text {Nis }}$ and using Theorem 7.2.1 (1), we get an isomorphism

$$
R \Gamma_{M}^{\downarrow} \circ R \underline{b}_{s}^{\mathrm{Nis}} \stackrel{\sim}{\rightarrow} R \underline{\Gamma}_{M}
$$

which yields the second isomorphism of Proposition 7.3.2.

\subsection{From $D(\underline{\mathbf{M N S}})$ to $D(\underline{\mathrm{MNST}})$}

Notation 7.4.1. Let $N \in \underline{\mathbf{M S m}}^{\text {fin }}$ and let $K$ be a complex on $\underline{\mathrm{MNST}}^{\text {fin }}$. We write $K_{N}$ for $\left(\underline{b}_{s}^{\mathrm{Nis}} \underline{c}^{\mathrm{Nis}} K\right)_{N}$.

The following extends Proposition 2.2.9 to unbounded complexes of sheaves.

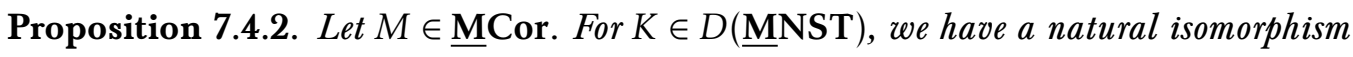

$$
\operatorname{Hom}_{D(\underline{\mathbf{M N S T}})}\left(\mathbb{Z}_{\mathrm{tr}}(M), K[i]\right) \simeq \underset{N \in \underline{\Sigma}^{\mathrm{fin}} \downarrow M}{\lim } \mathbb{H}_{\mathrm{Nis}}^{i}\left(\bar{N}, K_{N}\right) \simeq \underset{N \in \underline{\Sigma}_{\mathrm{fin}} \downarrow M}{\lim _{\mathrm{Nis}}} \mathbb{H}^{i}\left(\bar{N},\left(R \underline{b}_{s}^{\mathrm{Nis}} D\left(\underline{c}^{\mathrm{Nis}}\right) K\right)_{N}\right), \quad i \in \mathbb{Z} .
$$

Proof. Define as before functors $\underline{\Gamma}_{M}: \underline{\text { MNS }} \rightarrow$ Ab and $\underline{\Gamma}_{M}^{T}: \underline{\text { MNST }} \rightarrow$ Ab by

$$
\underline{\Gamma}_{M}(F)=F(M), \quad \underline{\Gamma}_{M}^{T}(G)=G(M) .
$$

We have $\underline{\Gamma}_{M}^{T}=\underline{\Gamma}_{M} \circ \underline{c}^{\mathrm{Nis}}$ thanks to the adjunction $\left(\underline{c}_{\mathrm{Nis}}, \underline{c}^{\mathrm{Nis}}\right)$ of Theorem 2.2.7. Moreover, $\underline{c}^{\mathrm{Nis}}$ is exact by this theorem. Let us show as before that the natural transformation (A.2.1)

$$
R \underline{\Gamma}_{M}^{T} \Rightarrow R \underline{\Gamma}_{M} \circ D\left(\underline{c}^{\mathrm{Nis}}\right)
$$

is invertible. We copy the argument of the previous subsection, using Lemma A.2.7.

Its first condition is given by [KMSY21, Lemma 4.6.1], which says that $\underline{c}^{\mathrm{Nis}}$ sends injectives to flabbys; by [KMSY21, Theorem A.9.1 and Lemma A.9.3], this already yields isomorphisms

$$
R^{p} \underline{\Gamma}_{M}^{T} \stackrel{\sim}{\rightarrow} R^{p} \underline{\Gamma}_{M} \circ \underline{c}^{\text {Nis }}, \quad p \geq 0 .
$$

We are now left to show the strong additivity of the three functors. For $D\left(\underline{c}^{\mathrm{Nis}}\right)$, this follows from the strong additivity of $\underline{c}^{\text {Nis }}$ (Theorem 2.2.7) and Proposition A.2.8 a). For the two other functors, we need to check the conditions of Proposition A.2.8 b); this was done for $R \underline{\Gamma}_{M}$ in the previous section, and the case of $R \underline{\Gamma}_{M}^{T}$ is reduced to this one by (7.4.2).

Thus we get an isomorphism

$$
\operatorname{Hom}_{D(\underline{\mathbf{M N S T}})}\left(\mathbb{Z}_{\mathrm{tr}}(M), L[i]\right) \stackrel{\sim}{\rightarrow} \operatorname{Hom}_{D(\underline{\mathbf{M N S}})}\left(\mathbb{Z}_{\mathrm{tr}}(M), \underline{\underline{c}}^{\mathrm{Nis}} L[i]\right) \quad i \in \mathbb{Z}
$$

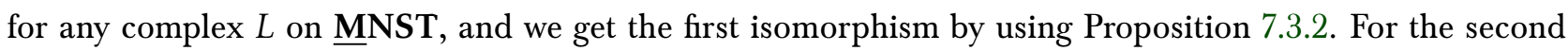
one, composing (7.3.3) with (7.4.1), we obtain an isomorphism

$$
R \underline{\Gamma}_{M}^{T} \simeq R \Gamma_{M}^{\downarrow} \circ R \underline{b}_{s}^{\mathrm{Nis}} \circ D\left(\underline{c}^{\mathrm{Nis}}\right)
$$

which yields the second isomorphism of Proposition 7.4.2. 


\subsection{From $D(\underline{\text { MNST }})$ to $D($ MNST)}

\section{Theorem 7.5.1.}

(1) The functor $D\left(\tau_{\mathrm{Nis}}\right): D(\mathbf{M N S T}) \rightarrow D(\underline{\text { MNST }})$ is fully faithful.

(2) One has isomorphisms

$$
\begin{aligned}
\operatorname{Hom}_{D(\mathbf{M N S T})}\left(\mathbb{Z}_{\mathrm{tr}}(M), K[i]\right) & \simeq \underset{N \in \underline{\Sigma}^{\mathrm{fin}} \downarrow M}{\lim } \mathbb{H}_{\mathrm{Nis}}^{i}\left(\bar{N}, K_{N}\right) \\
& \simeq \underset{N \in \underline{\Sigma}^{\mathrm{inin}} \downarrow M}{\lim } \mathbb{H}_{\mathrm{Nis}}^{i}\left(\bar{N},\left(R \underline{b}_{s}^{\mathrm{Nis}} D\left(\underline{c}^{\mathrm{Nis}} \tau_{\mathrm{Nis}}\right)\right) K_{N}\right), \quad i \in \mathbb{Z}
\end{aligned}
$$

for any complex $K$ on MNST, where $K_{N}:=\left(\underline{b}_{s}^{\mathrm{Nis}} \underline{c}^{\mathrm{Nis}} \tau^{\mathrm{Nis}} K\right)_{N}$.

Proof. Recall that $\tau_{\mathrm{Nis}}$ and $\tau^{\mathrm{Nis}}$ are both exact and strongly additive: see Lemma 4.2 .5 (2) and Theorem 5.1.1 ( $\tau_{\mathrm{Nis}}$ is strongly additive as a left adjoint). Then we get from Proposition A.2.4 an adjunction $\left(D\left(\tau_{\mathrm{Nis}}\right), D\left(\tau^{\mathrm{Nis}}\right)\right)$ and from Lemma A.2.7 an isomorphism

$$
D\left(\tau^{\mathrm{Nis}}\right) D\left(\tau_{\mathrm{Nis}}\right) \stackrel{\sim}{\leftarrow} D\left(\tau^{\mathrm{Nis}} \tau_{\mathrm{Nis}}\right) \stackrel{\sim}{\rightarrow} D(\mathrm{Id})=\mathrm{Id}
$$

which shows the full faithfulness of $D\left(\tau_{\mathrm{Nis}}\right)$. This shows (1). (2) now follows from (1) and Proposition 7.4.2 (2).

Remark 7.5.2. One can show that the essential image of $D\left(\tau_{\mathrm{Nis}}\right)$ is

$$
D_{\text {MNST }}(\underline{\text { MNST }}):=\left\{K \in D(\underline{\text { MNST }}) \mid H^{i}(K) \in \tau_{\text {Nis }}(\text { MNST }) \forall i \in \mathbb{Z}\right\} .
$$

Since the proof involves delicate and lengthy arguments relying on the notion of left-completeness, we skip it (see [KSY15]).

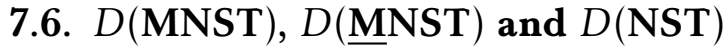

We leave it to the reader to produce an unbounded version of Theorem 6.3.2.

\section{Appendix: Categorical toolbox, II}

\section{A.1. A spectral sequence}

The following convenient proposition is used several times in the paper.

Proposition A.1.1. Let $a: \mathcal{B} \leftrightarrows \mathcal{A}: i$ be a pair of adjoint functors between abelian categories (a is left adjoint to i). Suppose that $\mathcal{A}$ has enough injectives and that a is exact. Then, for any $(A, B) \in \mathcal{A} \times \mathcal{B}$, there is a convergent spectral sequence

$$
\operatorname{Ext}_{\mathcal{B}}^{p}\left(B, R^{q} i A\right) \Rightarrow \operatorname{Ext}_{\mathcal{A}}^{p+q}(a B, A)
$$

If $B$ is projective, this spectral sequence collapses to isomorphisms

$$
\mathcal{B}\left(B, R^{q} i A\right) \simeq \operatorname{Ext}_{\mathcal{A}}^{q}(a B, A) .
$$

Proof. Fix B. By adjunction, the composition of functors

$$
\mathcal{A} \stackrel{i}{\rightarrow} \mathcal{B} \stackrel{\mathcal{B}(B,-)}{\longrightarrow} \mathbf{A b}
$$

is isomorphic to $\mathcal{A}(a B,-)$. We then get the spectral sequence from [KMSY21, Theorem A.9.1, Example A.9.2]. The last fact is obvious. 


\section{A.2. Unbounded derived categories}

Theorem A.2.1 ([KS06, Theorem 14.3.1]). Let $\mathcal{A}$ be a Grothendieck category.

a) Let $K(\mathcal{A})$ be the unbounded homotopy category of $\mathcal{A}$. The localisation functor $\lambda_{\mathcal{A}}: K(\mathcal{A}) \rightarrow D(\mathcal{A})$ has a right adjoint $\rho_{\mathcal{A}}$, whose essential image is (by definition) the full subcategory of homotopically injective complexes. $^{2}$

b) Let $F: K(\mathcal{A}) \rightarrow \mathcal{T}$ be a triangulated functor. Then $F$ has a (universal) right Kan extension $R F$ relative to $\lambda_{\mathcal{A}}$, given by $R F=F \circ \rho_{\mathcal{A}}$. In particular, any left exact functor $F: \mathcal{A} \rightarrow \mathcal{B}$, where $\mathcal{B}$ is another abelian category, has a total right derived functor $R F: D(\mathcal{A}) \rightarrow D(\mathcal{B})$ given by $R F=\lambda_{\mathcal{B}} \circ K(F) \circ \rho_{\mathcal{A}}$.

c) The restriction of $R F$ to $D^{+}(\mathcal{A})$ is the total derived functor $R^{+} F$ (cf. [Ver77, §2, Remark 1.6]).

Let us justify c), which is not in [KS06]: the point is that $\rho_{\mathcal{A}}$ carries $D^{+}(\mathcal{A})$ into $K^{+}(\mathcal{A})[\mathrm{KS} 06$, Theorem 13.3.7].

Definition A.2.2. Let $F: \mathcal{A} \rightarrow \mathcal{B}$ be a left exact functor between Grothendieck categories. An object $C \in K(\mathcal{A})$ is $F$-acyclic if the morphism

$$
\lambda_{\mathcal{B}} K(F) C \rightarrow R F \lambda_{\mathcal{A}} C=\lambda_{\mathcal{B}} K(F) \rho_{\mathcal{A}} \lambda_{\mathcal{A}} C
$$

given by the unit map of the adjunction $\left(\lambda_{\mathcal{A}}, \rho_{\mathcal{A}}\right)$ is an isomorphism.

Example A.2.3. If $F$ is exact, every object of $K(\mathcal{A})$ is $F$-acyclic.

Let $\mathcal{A} \stackrel{F}{\rightarrow} \mathcal{B} \stackrel{G}{\rightarrow} \mathcal{C}$ be a chain of left exact functors between Grothendieck categories. The unit map of the adjunction $\left(\lambda_{\mathcal{B}}, \rho_{\mathcal{B}}\right)$ yields a natural transformation

$$
R(G F) \Rightarrow R G \circ R F \text {. }
$$

The following lemma is made tautological by Definition A.2.2:

Lemma A.2.4. (A.2.1) is a natural isomorphism if and only if F carries homotopically injectives to G-acyclics. In particular, (A.2.1) is a natural isomorphism provided $G$ is exact (see Example A.2.3).

Here is a first application:

Proposition A.2.5. Assume $\mathcal{C}=\mathcal{A}, F$ right adjoint to $G$, and $G$ exact. Then $R F$ is right adjoint to $R G=D(G)$.

Proof. This is a special case of [KS06, Theorem 14.4.5].

We come back to the general situation. Suppose that $F$ carries injectives of $\mathcal{A}$ to $G$-acyclics. Then [KMSY21, Theorem A.9.1] implies that (A.2.1) is an isomorphism when restricted to $D^{+}(\mathcal{A})$ ([Ver77, §2, Proposition 3.1], [KS06, Theorem 13.3.7 and Proposition 13.3.13]). This is not true on $D(\mathcal{A})$ in general, as pointed out by Ayoub and Riou:

Example A.2.6. Let $\mathcal{B}=\operatorname{Mod}-\mathbb{Z}[\mathbb{Z} / 2], \mathcal{A}=\mathcal{B}^{\mathbb{N}}$ and $\mathcal{C}=\mathbf{A b}$; let $F=\bigoplus_{\mathbb{N}}$ and $G=H^{0}(\mathbb{Z} / 2,-)$. The above hypotheses are verified: since $\mathbb{Z}[\mathbb{Z} / 2]$ is Noetherian, a direct sum of injectives is injective. Let $M=(\mathbb{Z} / 2[n])_{n \in \mathbb{N}} \in D(\mathcal{A})$. We claim that the map

$$
R(G F)(M) \rightarrow R G \circ R F(M)
$$

is not an isomorphism. Indeed, $G F=F^{\prime} G^{\prime}$ where $G^{\prime}: \mathcal{A} \rightarrow \mathbf{A b}^{\mathbb{N}}$ is $H^{0}(\mathbb{Z} / 2,-)$ and $F^{\prime}: \mathbf{A} \mathbf{b}^{\mathbb{N}} \rightarrow \mathbf{A b}$ is $\bigoplus_{\mathbb{N}}$. Let $C=R G(\mathbb{Z} / 2)$, so that $H^{q}(C)=\mathbb{Z} / 2$ for $q \geq 0$ and $H^{q}(C)=0$ for $q<0$. Then, by Lemma A.2.4:

$$
R(G F)(M)=R\left(F^{\prime} G^{\prime}\right)(M)=R F^{\prime} \circ R G^{\prime}(M)=\bigoplus_{n \in \mathbb{N}} C[n] .
$$

\footnotetext{
${ }^{2}$ This is the same notion as Spaltenstein's $K$-injective [Spa88].
} 
On the other hand,

$$
R G \circ R F(M)=R G\left(\bigoplus_{n \in \mathbb{N}} \mathbb{Z} / 2[n]\right)
$$

But, in $D(\mathcal{B})$, we have $\bigoplus_{n \in \mathbb{N}} \mathbb{Z} / 2[n] \stackrel{\sim}{\rightarrow} \prod_{n \in \mathbb{N}} \mathbb{Z} / 2[n]$, and $R G$ commutes with products as a right adjoint. Hence

$$
R G \circ R F(M)=\prod_{n \in \mathbb{N}} C[n] .
$$

For $q \in \mathbb{Z}$, we have $H^{q}\left(\bigoplus_{n \in \mathbb{N}} C[n]\right)=\bigoplus_{q+n \geq 0} \mathbb{Z} / 2$ and $H^{q}\left(\prod_{n \in \mathbb{N}} C[n]\right)=\prod_{q+n \geq 0} \mathbb{Z} / 2$.

However, we have the following lemma of Ayoub:

Lemma A.2.7. Suppose that $F$ carries injectives to $G$-acyclics and that $R F, R G$ and $R(G F)$ are strongly additive. Then (A.2.1) is an isomorphism.

(In example A.2.6, $R G$ is not strongly additive.)

Proof (Ayoub). Let $M \in D(\mathcal{A})$. We have to show that (A.2.2) is an isomorphism. Viewing $M$ as an object of $K(\mathcal{A})$, we have an isomorphism

$$
\operatorname{hocolim}_{n} \sigma_{\geq n} M \stackrel{\sim}{\rightarrow} M
$$

where $\sigma_{\geq n}$ is the stupid truncation. This isomorphism still holds in $D(\mathcal{A})$, because $\lambda_{\mathcal{A}}$ is strongly additive. By the hypothesis, this reduces us to the case where $M \in D^{+}(\mathcal{A})$, and therefore to Grothendieck's theorem (cf. Theorem A.2.1 c)).

Let $F: \mathcal{A} \rightarrow \mathcal{B}$ be a left exact functor between Grothendieck categories. In view of Lemma A.2.7, we need a practical sufficient condition to ensure that $R F$ is strongly additive. The following ones are adapted to the context of this paper:

\section{Proposition A.2.8.}

a) If $F$ is strongly additive and exact, $R F=D(F)$ is strongly additive.

b) Suppose that

(i) For any $p \geq 0, R^{p} F$ is strongly additive.

(ii) There exists a set $\mathcal{E}$ of compact projective generators of $\mathcal{B}$ such that, for any $E \in \mathcal{E}$, there is an integer $c d_{F}(E)$ such that

$$
\mathcal{B}\left(E, R^{p} F(A)\right)=0 \text { for } p>c d_{F}(E) \text { and for all } A \in \mathcal{A} .
$$

Then RF is strongly additive.

c) Suppose that $R F$ admits a left adjoint $G$ which sends a set $\left(E_{\alpha}\right)$ of compact generators of $D(\mathcal{B})$ to compact objects of $D(\mathcal{A})$. Then $R F$ is strongly additive.

Proof. a) The strong additivity of $F$ easily implies that of $K(F)$, which in turn implies that of $D(F)$ since $\lambda_{\mathcal{B}}$ is strongly additive as a left adjoint.

b) Let $\left(C_{i}\right)_{i \in I} \in D(\mathcal{A})^{I}$. We must show that the map

$$
\bigoplus_{i \in I} R F\left(C_{i}\right) \rightarrow R F\left(\bigoplus_{i \in I} C_{i}\right)
$$

is an isomorphism. Since the $E[n], E \in \mathcal{E}, n \in \mathbb{Z}$, are a set of generators of $D(\mathcal{B})$, it suffices to check this after applying $D(\mathcal{B})(E[n],-)$ for all $(E, n)$. Since $E$ is projective, we have an isomorphism

$$
D(\mathcal{B})(E[n], D) \simeq \mathcal{B}\left(E, H^{n}(D)\right)
$$


for any $D \in D(\mathcal{B})$; since $E$ is compact in $\mathcal{B}$, this formula shows that $E[n]$ is compact in $D(\mathcal{B})$. Therefore we must show that the homomorphisms

$$
\bigoplus_{i \in I} \mathcal{B}\left(E, H^{n}\left(R F C_{i}\right)\right) \rightarrow \mathcal{B}\left(E, H^{n}\left(R F \bigoplus_{i \in I} C_{i}\right)\right)
$$

are bijective. By (ii), the spectral sequence

$$
\mathcal{B}\left(E, R^{p} F H^{q}(C)\right) \Rightarrow \mathcal{B}\left(E, H^{p+q}(R F C)\right)
$$

converges for any $C \in D(\mathcal{A})$. Thus it suffices to show that the homomorphisms

$$
\bigoplus_{i \in I} \mathcal{B}\left(E, R^{p} F H^{q}\left(C_{i}\right)\right) \rightarrow \mathcal{B}\left(E, R^{p} F H^{q}\left(\bigoplus_{i \in I} C_{i}\right)\right)
$$

are bijective. By (i), this follows from the compactness of $E$.

c) Keep the notation of b). We may test (A.2.3) on the $E_{\alpha}$ 's. By their compactness, we must show that the composition

$$
\bigoplus_{i \in I} D(\mathcal{B})\left(E_{\alpha}, R F\left(C_{i}\right)[q]\right) \rightarrow D(\mathcal{B})\left(E_{\alpha}, \bigoplus_{i \in I} R F\left(C_{i}\right)[q]\right) \rightarrow D(\mathcal{B})\left(E_{\alpha}, R F\left(\bigoplus_{i \in I} C_{i}\right)[q]\right)
$$

is an isomorphism for all $q \in \mathbb{Z}$. By adjunction, it is transformed into

$$
\bigoplus_{i \in I} D(\mathcal{A})\left(G\left(E_{\alpha}\right), C_{i}[q]\right) \rightarrow D(\mathcal{A})\left(G\left(E_{\alpha}\right), \bigoplus_{i \in I} C_{i}[q]\right)
$$

which is an isomorphism since the $G\left(E_{\alpha}\right)$ are compact.

Finally, we need a practical sufficient condition to ensure that, in Condition (i) of Proposition A.2.8 b), the case $p=0$ implies the cases $p>0$. This is given by the classical

Lemma A.2.9. Suppose that $F$ is strongly additive and that, in $\mathcal{A}$, infinite direct sums of injectives are $F$-acyclic. Then $R^{p} F$ is strongly additive for any $p>0$.

Proof. Décalage.

\section{References}

[KM18] B. Kahn and H. Miyazaki, Mayer-Vietoris triangles for motives with modulus, preprint arXiv:1809.05851 (2018).

[KM20] T Topologies on schemes and modulus pairs, Nagoya Math. J., to appear. http://dx.doi.org/10.1017/nmj.2020.15

[KMSY20] B. Kahn, H. Miyazaki, S. Saito, and T. Yamazaki, Motives with modulus, III: The categories of motives, preprint arXiv:2011.11859 (2020).

[KMSY21] _ Motives with modulus, I: Modulus sheaves with transfers for non proper modulus pairs, Épijournal de Géométrie Algébrique 5 (2021), article 1.

[KS06] M. Kashiwara and P. Schapira, Categories and sheaves, Springer-Verlag, Berlin, 2006.

[KSY15] B. Kahn, S. Saito, and T. Yamazaki Motives with modulus, preprint arXiv:1511.07124 (2015), withdrawn.

[Mac98] S. Mac Lane, Categories for the working mathematician. Second edition. Graduate Texts in Mathematics, vol. 5, Springer-Verlag, New York, 1998. 
[MVW06] C. Mazza, V. Voevodsky, and C. Weibel, Lecture notes on motivic cohomology, Clay Mathematics Monographs, vol. 2, American Mathematical Society, Providence, RI; Clay Mathematics Institute, Cambridge, MA, 2006.

[Miy19] H. Miyazaki, Cube invariance of higher Chow groups with modulus, J. Algebraic Geom. 28 (2019), no. 2, 339-390.

[Miy20] H. Miyazaki, Nisnevich topology with modulus, Ann. K-Theory 5 (2020), no. 3, 581-604.

[RG71] M. Raynaud and L. Gruson, Critères de platitude et de projectivité. Techniques de "platification" d'un module, Invent. Math. 13 (1971), 1-89.

[SGA4] Théorie des topos et cohomologie étale des schémas. Séminaire de Géométrie Algébrique du Bois-Marie 1963-1964 (SGA 4). Dirigé par M. Artin, A. Grothendieck et J. L. Verdier. Lecture Notes in Math., vol. 269, 270, 305, Springer-Verlag, Berlin-New York, 1972-73.

[Spa88] N. Spaltenstein, Resolutions of unbounded complexes, Compositio Math. 65 (1988), no. 2, 121-154.

[Ver77] J.-L. Verdier, Catégories dérivées, quelques résultats (état 0). In: Cohomologie étale (SGA 4 1/2), pp. 262-311, Lecture Notes in Math., vol. 569, Springer, Berlin, 1977.

[Voe00] V. Voevodsky, Triangulated categories of motives over a field. In: Cycles, transfers, and motivic cohomology theories, pp. 188-238, Ann. Math. Studies, vol. 143, Princeton University Press, 2000.

[Voe10a] _ Homotopy theory of simplicial sheaves in completely decomposable topologies, J. Pure Appl. Algebra 214 (2010), no. 8, 1384-1398.

[Voe10b] _ Unstable motivic homotopy categories in Nisnevich and cdh-topologies, J. Pure Appl. Algebra 214 (2010), no. 8, 1399-1406. 